\title{
JOININGS OF HIGHER RANK DIAGONALIZABLE ACTIONS ON LOCALLY HOMOGENEOUS SPACES
}

\author{
MANFRED EINSIEDLER AND ELON LINDENSTRAUSS
}

\begin{abstract}
We classify joinings between a fairly general class of higher rank diagonalizable actions on locally homogeneous spaces. In particular, we classify joinings of the action of a maximal $\mathbb{R}$-split torus on $G / \Gamma$, with $G$ a simple Lie group of $\mathbb{R}$-rank $\geq 2$ and $\Gamma<G$ a lattice. We deduce from this a classification of measurable factors of such actions, as well as certain equidistribution properties.
\end{abstract}

\section{INTRODUCTION}

In a landmark paper [9] Furstenberg introduced two fundamental ideas: 1) joinings, and how they can be used to investigate dynamical systems both in the topological and the measurable category 2) for natural multidimensional algebraic actions (Furstenberg studies the example of multiplication by multiplicatively independent integers $p$ and by $q$ on the one torus), it is often the case that while the action of every individual element is quite flexible, the full action may display remarkable rigidity properties. Both of these ideas have had a major impact on modern ergodic theory. Joinings and the related notion of disjointness have become an indispensable tool to ergodic theorists (to illustrate this point, one may look for example at [10]). The multidimensional algebraic action whose study Furstenberg pioneered still contain many mysteries and are far from being understood, but substantial progress has been made and this study, particularly in the context of locally homogeneous spaces such as $\mathrm{SL}(n, \mathbb{R}) / \mathrm{SL}(n, \mathbb{Z})$, has found applications in number theory and the arithmetic theory of quantum chaos (which is closely connected to the theory automorphic forms); we refer the reader to the survey [21] or to $[20,6]$ for more details. It is interesting to note

Date: July 2005.

M.E. acknowledges support of NSF Grant 0400587 and 0509350. This research was partially conducted during the period M. E. was employed by the Clay Mathematics Institute as a Research Scholar. E.L. acknowledges the support of Clay Mathematics Institute in the form of a Clay Research Fellowship and NSF Grant DMS-0434403. 
that the first such application was provided already by Furstenberg in his original paper [9].

In this paper we consider the following type of algebraic dynamical systems: we take $G$ to be a connected Lie group, $\Gamma<G$ a lattice, i.e. a discrete group of finite covolume, and let $\alpha: \mathbb{R}^{n} \rightarrow G$ be an injective homomorphism to an $\mathbb{R}$-split torus of $G$ (more precisely what we need is that the image of $\alpha$ composed with the adjoint representation of $G$ is diagonalizable over $\mathbb{R}$ ). Then $\alpha$ gives us an action of $\mathbb{R}^{n}$ on $G / \Gamma$ by left translations, and in this paper we will always assume that $n \geq 2$.

Such systems are natural generalizations of the system considered by Furstenberg of multiplication by $p$ and by $q$ on the one torus. Here again the properties of the action of any individual element is very different from that of the full group. For example, there are many invariant measures for the action of any individual element or even every one dimensional (connected) subgroup of $\mathbb{R}^{n}$. For the full action, however, Furstenberg, Katok and Spatzier [14] and Margulis [24] conjectured that $\alpha$-invariant probability measures are rare: they are all algebraic unless there are factors for which the actions degenerates to a rank one situation, where we call a probability measure algebraic if it is an $L$-invariant measure on a single (necessarily closed) orbit of a closed subgroup $L \leq G$.

Progress on that conjecture has been made so far only under additional assumptions $[14,15,5,12]$. In some cases [29, 7, 20, 6] the only remaining additional assumption is that the measure has positive entropy under some element of the action.

We combine the two themes in Furstenberg's original paper by studying joinings of two such systems. For $i=1,2$ let $\alpha_{i}$ be an actions of $\mathbb{R}^{n}$ as above on $X_{i}=G_{i} / \Gamma$ and let $m_{i}$ be the normalized Haar measure on $X_{i}$. Define $\alpha^{\mathbf{t}}=\alpha_{1}^{\mathbf{t}} \times \alpha_{2}^{\mathbf{t}}$ for $\mathbf{t} \in \mathbb{R}^{n}$. A joining between $\alpha_{1}$ and $\alpha_{2}$ (with respect to $m_{1}$ and $m_{2}$ ) is an $\alpha$-invariant measure $\mu$ on $X=X_{1} \times X_{2}$ such that $\left(\pi_{i}\right)_{*} \mu=m_{i}$ for $i=1,2$.

Before we give our theorem we give some examples of joinings. The trivial joining $m_{1} \times m_{2}$ always exists. A more general class of joinings are algebraic joinings: Suppose $H \subset G_{1} \times G_{2}$ contains $\alpha\left(\mathbb{R}^{n}\right)$ and projects surjectively to $G_{i}$ for $i=1,2$. If $H\left(x_{1}, x_{2}\right)$ is closed with finite volume for some $\left(x_{1}, x_{2}\right) \in X_{1} \times X_{2}$, then its (normalized) volume measure $\mu$ gives an algebraic joining between $\alpha_{1}$ and $\alpha_{2}$.

The actions $\alpha_{1}$ and $\alpha_{2}$ are disjoint if the trivial joining is the only joining between them.

Note that if $n=1$, the time one maps $\alpha_{i}^{1}$ have positive entropy (and are even Bernoulli automorphisms [2]). Therefore, in this case $\alpha_{1}^{1}$ and $\alpha_{2}^{1}$ have for any small enough $s$ common Bernoulli factors of 
entropy $s$ by Sinai's factor theorem (see [30] or [10, Ch. 20]). The well known construction of the relatively independent joining over a factor (see (6.4)) gives now uncountably many non-algebraic joinings. This explains the requirement of injectivity and $n \geq 2$.

In this paper we give an unconditional classification of joinings for such $\mathbb{R}^{n}$-actions in many cases. In particular, we prove the following:

Theorem 1.1. For $i=1,2$ let $G_{i}$ be a connected simple Lie group, let $\Gamma_{i} \subset G_{i}$ be a lattice, let $m_{i}$ be the normalized Haar measure on $X_{i}=G_{i} / \Gamma_{i}$, and let $\alpha_{i}: \mathbb{R}^{n} \rightarrow G_{i}$ be an injective homomorphism to an $\mathbb{R}$-split torus of $G_{i}$. Suppose $n \geq 2$ and that $\mu$ is an ergodic joining between $\alpha_{1}$ and $\alpha_{2}$, then $\mu$ is algebraic. Moreover, if $\mu$ is not the trivial joining, then $G_{1}$ and $G_{2}$ are locally isomorphic and the lattices induced by $\Gamma_{1}$ and $\Gamma_{2}$ in the common universal cover $G$ of $G_{1}$ and $G_{2}$ are commensurable up to an automorphism of $G$ intertwining the actions corresponding to $\alpha_{1}$ and $\alpha_{2}$.

Recall that two lattices $\Gamma_{1}$ and $\Gamma_{2}$ in a Lie group $G$ are commensurable if $\Gamma_{1} \cap \Gamma_{2}$ has finite index in $\Gamma_{1}$ and $\Gamma_{2}$ (or equivalently if $\Gamma_{1} \cap \Gamma_{2}$ is still a lattice).

With respect to the assumption of ergodicity in Theorem 1.1 note that any joining $\mu$ can be decomposed into ergodic components with respect to $\alpha$ (see [32] or [11, Thm. 4.2.6]), and that the projection of this decomposition to $X_{i}$ gives a decomposition of $m_{i}$. However, since the latter is an ergodic measure for the action $\alpha_{i}$ by Moore's ergodic criterion (see [25] or Section 2.2 for more details) it follows that a.e. ergodic component of $\mu$ is an ergodic joining. Therefore, the characterization of ergodic joinings in Theorem 1.1 gives a complete description of all possible joinings between these actions.

We remark that for the dynamical system considered by Furstenberg of multiplication by $p$ and $q$ or more generally actions of non virtuallycyclic groups of commuting toral endomorphisms a full classifications of joinings has not been obtained, the available results describe ergodic joinings only up to zero entropy $[13,12,7]$. In fact, for these actions the classifications of joinings is equivalent to the classifications of all invariant measures, which is a well known open problem.

From Theorem 1.1 we easily obtain using a standard argument (originally due to Furstenberg) the following consequence about equidistribution of orbits. Let $\alpha$ be an $\mathbb{R}^{n}$-action on a locally compact metric space $X$, let $\mu$ be an $\alpha$-ergodic and invariant probability measure on $X$. We say that a point $x \in X$ has $\mu$-equidistributed $\alpha$-orbit if

$$
\lim _{\ell \rightarrow \infty} \frac{1}{(2 \ell)^{n}} \int_{[-\ell, \ell]^{n}} f\left(\alpha^{\mathbf{t}} x\right) \mathrm{d} \mathbf{t}=\int f \mathrm{~d} \mu
$$


for all $f \in C(X)$ with compact support. ${ }^{1}$ Note that for any ergodic $\mu$, one has that $\mu$-a.e. point has $\mu$-equidistributed $\alpha$-orbit by the pointwise ergodic theorem.

Corollary 1.2. Let $G$ be a simple connected Lie group and let $\alpha: \mathbb{R}^{n} \rightarrow$ $G$ be an injective homomorphism to an $\mathbb{R}$-split torus of $G$. For $i=1,2$ let $\Gamma_{i} \subset G$ be a lattice and let $m_{i}$ be the normalized Haar measure on $X_{i}=G / \Gamma_{i}$. Suppose $n \geq 2$ and that there is no automorphism of $G$ commuting with left translations by elements in the image of $\alpha$ taking $\Gamma_{2}$ to a lattice commensurable with $\Gamma_{1}$. If $x_{i} \in X_{i}$ has $m_{i}$-equidistributed $\alpha$-orbit for $i=1,2$, then $\left(x_{1}, x_{2}\right) \in X_{1} \times X_{2}$ has $m_{1} \times m_{2}$-equidistributed $\alpha$-orbit, where $\alpha$ acts on $X_{1} \times X_{2}$ by left multiplication with $\alpha^{\mathbf{t}} \times \alpha^{\mathbf{t}}$ for $\mathbf{t} \in \mathbb{R}^{n}$.

Clearly, a similar statement holds if $X_{i}=G_{i} / \Gamma_{i}$ for two locally nonisomorphic simple connected Lie groups $G_{1}$ and $G_{2}$.

We now describe a more general setup that allows us to show rigidity of joinings. We will not assume that $G$ is simple, or even semisimple. Note however, that if for instance

$$
X=\mathrm{SL}(2, \mathbb{R}) \times \mathrm{SL}(2, \mathbb{R}) / \mathrm{SL}(2, \mathbb{Z}) \times \mathrm{SL}(2, \mathbb{Z})
$$

then the projection to the first coordinate is well defined and the $\mathbb{R}^{2}$ action on $X$ descends to $Y=\mathrm{SL}(2, \mathbb{R}) / \mathrm{SL}(2, \mathbb{Z})$. However, in this factor the subgroup $\{0\} \times \mathbb{R}$ acts trivially $-Y$ is a (global) rank one factor. Similar to the case of $n=1$ the action $\alpha$ on $X$ allows many nonalgebraic joinings. We avoid this situation with the following definition.

Definition 1.3. Let $G$ be a Lie group and let $\alpha: \mathbb{R}^{n} \rightarrow G$ be a homomorphism. We say $\alpha$ is $\mathbb{R}$-diagonalizable if the associated adjoint action of $\alpha$ on the Lie algebra $\mathfrak{g}$ is diagonalizable over $\mathbb{R}$. If $\mathfrak{h} \subset \mathfrak{g}$ is a Lie ideal, then consider the induced action of $\alpha$ via the adjoint representation on $\mathfrak{g} / \mathfrak{h}$. We say that $\alpha$ has no local rank one factors if for any proper Lie ideal $\mathfrak{h}$ the weights of the adjoint action of $\alpha$ on $\mathfrak{g} / \mathfrak{h}$ do not lie on a one-dimensional subspace.

In particular, if $\alpha$ is an $\mathbb{R}^{n}$-action with no local rank one factors then $n>1$. Conversely if $G$ is a simple Lie group, $n>1, \alpha$ is injective and $\mathbb{R}$-diagonalizable, then $\alpha$ has no local rank one factors. To see this note first that the only proper Lie ideal is $\mathfrak{h}=\{0\}$. However, for $\mathfrak{g}$ the Lyapunov weights of $\alpha$ cannot lie in a one-dimensional subspace, otherwise there exists some $\mathbf{t}$ with $\lambda(\mathbf{t})=0$ for all Lyapunov weights and

\footnotetext{
${ }^{1}$ One can replace the cube $[-\ell, \ell]^{n}$ with other averaging sets, e.g. balls around 0 or $[0, \ell]^{n}$. The latter gives a weaker notion of equidistribution, but the above remains true also in this case.
} 
$\alpha^{\mathbf{t}}$ would belong to the center of $G$. Therefore the following generalizes Theorem 1.1

Theorem 1.4. For $i=1,2$ let $G_{i}$ be a connected Lie group, let $\Gamma_{i}$ be a lattice in $G_{i}$, and let $m_{i}$ be the Haar measure on $X_{i}=G_{i} / \Gamma_{i}$. Furthermore, let $\alpha_{i}: \mathbb{R}^{n} \rightarrow G_{i}$ be injective homomorphism that are $\mathbb{R}$ diagonalizable and have no local rank one factors. Let $\mu$ be an ergodic joining between $\alpha_{1}$ and $\alpha_{2}$. Then there is a closed subgroup $H \leq G_{1} \times G_{2}$ projecting on to $G_{1}$ and $G_{2}$ such that $\mu$ is the Haar measure on a closed $H$-orbit in $X_{1} \times X_{2}$.

It is a general fact due to Dave Witte Morris [34, 35] that an algebraic classification of self joinings of the $\mathbb{R}^{n}$-action given by $\alpha$ on $(G / \Gamma, m)$ can be used to classify measurable factors of this action, or equivalently countably generated $\alpha$ invariant $\sigma$-algebras of Borel subsets of $G / \Gamma$. A special case of this general principle was given earlier by Marina Ratner in [27]. Using this we can deduce the following from Theorem 1.4:

Theorem 1.5. Let $G$ be a connected Lie group, $\Gamma$ a lattice in $G$, and $m$ the Haar measure on $X=G / \Gamma$. Let $\alpha: \mathbb{R}^{n} \rightarrow G$ be an $\mathbb{R}$-diagonalizable injective homomorphism with no local rank one factors. Let $\mathcal{A}$ be an $\alpha$ invariant countably generated $\sigma$-algebra of Borel subsets of $G / \Gamma$. Then there is a surjective homomorphism of Lie groups $\psi: G \rightarrow G_{1}$, a lattice $\Gamma_{1} \geq \psi(\Gamma)$ (in particular, $\psi(\Gamma)$ is discrete), and a compact subgroup $\Phi$ of the group of affine automorphisms $\operatorname{Aff}\left(G_{1} / \Gamma_{1}\right)$ of $G_{1}$ modulo right translation by elements of $\Gamma_{1}$ so that $\mathcal{A}$ is equivalent mod $m$ to $\psi^{-1}\left(\mathcal{B}_{\Phi}\right)$, with $\mathcal{B}_{\Phi}$ denoting the $\sigma$-algebra of $\Phi$-invariant subsets of $G_{1} / \Gamma_{1}$.

(Note that since $\Phi$ is compact, $\mathcal{B}_{\Phi}$ and hence $\psi^{-1}\left(\mathcal{B}_{\Phi}\right)$ is automatically countably generated, see Corollary 6.7.)

In particular, as we will explicate in $\S 6$, Theorem 1.5 implies the following:

Corollary 1.6. Let $G$ be a connected semisimple Lie group with finite center and each factor of $G$ of $\mathbb{R}$-rank $\geq 2$, let $\Gamma<G$ be an irreducible lattice, and let $\alpha$ be an embedding of $\mathbb{R}^{n}$ onto the connected component of the identity of a maximal $\mathbb{R}$-split torus ${ }^{2}$. Let $M$ denote a maximal compact subgroup of $C_{G}\left(\alpha\left(\mathbb{R}^{n}\right)\right)$. Consider the action from the left given by $\alpha$ on the double quotient $X=M \backslash G / \Gamma$; this action preserves a smooth probability measure $m$. Let $\mathcal{A}$ be a nontrivial ${ }^{3} \alpha$-invariant

\footnotetext{
${ }^{2}$ In other words the image of $\alpha$ is precisely the closed connected subgroup $A$ appearing in the Iwasawa decomposition $K A N$ of $G$, equivalently the Lie algebra of $A$ is the negative eigenspace of a Cartan involution $\theta$ of a $\theta$-stable maximally non-compact Cartan subalgebra.

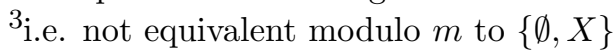


countably generated $\sigma$-algebra of Borel subsets of $X$. Then for $m$-a.e. $x \in X$ the atom $[x]_{\mathcal{A}}$ is finite. Moreover, if $\Gamma$ is maximal, and every automorphism of $G$ fixing $\alpha\left(\mathbb{R}^{n}\right)$ pointwise is an inner automorphism, then $\left|[x]_{\mathcal{A}}\right|=1$ a.s., in other words $\mathcal{A}$ is equivalent mod $m$ to the full Borel $\sigma$-algebra.

For example, any automorphism of $\operatorname{PGL}(n, \mathbb{R})$ fixing the positive diagonal matrices (which are the identity component of a maximal $\mathbb{R}$-split torus) is an inner automorphism.

Previous results on joinings of semisimple actions were obtained by Kalinin and Spatzier [12], but their method of proof required an additional assumption regarding ergodicity of certain one parameter subgroups on the joining $\mu$. Here we will use as the main tool the high entropy argument as provided in a recent work by A. Katok and the first named author [4]. Our argument also uses elements of [8], where disjointness results were obtained for actions on zero-dimensional compact abelian groups. These tools will lead us to a situation where we will be able to apply Ratner's theorem [28] for a subgroup of $G$ generated by unipotent elements.

More generally, we expect that similar results can be proved for e.g. semisimple Lie groups even if $G_{i}$ have local rank one factors, as long as the action of $\alpha_{i}$ on $G_{i} / \Gamma_{i}$ have no global rank one factors. This, however, will require a totally different proof, and in particular will require a general version of the low entropy argument used in [20, 6], a general version which we are currently in the process of writing.

\section{Definitions, ERgodicity, And Entropy}

In this section we summarize some background material needed for the proof of Theorem 1.4.

2.1. Lyapunov weights. We recall some definitions from [4]. Let $G$ be a connected Lie group with Lie algebra $\mathfrak{g}$ and let $\Gamma \subset G$ be a lattice. Recall that for $a \in G$ the derivative of the map $x \mapsto a x a^{-1}$ defines the adjoint automorphism $\operatorname{Ad}_{a}$ of $\mathfrak{g}$. Let $\alpha$ be an $\mathbb{R}^{n}$-action on $G / \Gamma$. Then $\alpha$ induces an adjoint action on $\mathfrak{g}$ of $G$, and $\alpha$ is Ad-semisimple if the adjoint action is diagonalizable over $\mathbb{C}$, and $\mathbb{R}$-diagonalizable if the same holds over $\mathbb{R}$. The logarithm of the absolute value of the eigenvalues of this action can be collected to form the Lyapunov weights, which are linear functionals $\lambda: \mathbb{R}^{n} \rightarrow \mathbb{R}$. For every Lyapunov weight $\lambda$ there corresponds a sum of eigenspaces $\mathfrak{u}^{\lambda}$ which is called the Lyapunov space corresponding to $\lambda$. 
Note that for an $\mathbb{R}$-diagonalizable action the Lyapunov weights are the weights of the adjoint representation restricted to $\alpha$ and the Lyapunov weight spaces are in fact the eigenspaces for the adjoint action. Moreover, if for some $\mathbf{t} \in \mathbb{R}^{n}$ all weights $\lambda(\mathbf{t})$ are zero, then $\alpha^{\mathbf{t}}$ is in the center of the group.

We define an equivalence relation between the Lyapunov weights, $\lambda \sim \eta$ if there is an $c>0$ so that $\lambda=c \eta$. For every nonzero equivalence class $\Lambda$ we define the coarse Lyapunov space $\mathfrak{u}^{\Lambda}$ by

$$
\mathfrak{u}^{\Lambda}=\bigoplus_{\lambda \in \Lambda} \mathfrak{u}^{\lambda}
$$

An element $u \in G$ is Ad-unipotent if $\operatorname{Ad}_{u}$ is unipotent as an automorphism of $\mathfrak{g}$, and a subgroup $U \leq G$ is Ad-unipotent if all of its elements are. The coarse Lyapunov spaces $\mathfrak{u}^{\Lambda}$ are in fact nilpotent Lie subalgebras, and the coarse Lyapunov subgroups $U^{\Lambda}=\exp \mathfrak{u}^{\Lambda}$ are connected, Ad-unipotent, and normalized by $\alpha$.

2.2. The Mautner phenomenon and ergodicity. The Mautner phenomenon (see [25], [31, Thm. 2.1], or [16, Thm. 2.1.4]) implies the following proposition.

Proposition 2.1. Let $G$ be a connected Lie group, let $\Gamma \subset G$ be a lattice, and let $\alpha$ be an $\mathbb{R}^{n}$-action without local rank one factors on the locally homogeneous space $X=G / \Gamma$. Then there exists a two dimensional subspace in $\mathbb{R}^{n}$ such that the restriction $\tilde{\alpha}$ of $\alpha$ to that plane still has no local rank one factors, and any nonzero element $\mathbf{t} \in \mathbb{R}^{2}$ satisfies that $a=\tilde{\alpha}^{\mathbf{t}} \in G$ acts ergodically with respect to the Haar measure $m$ of $X$.

Proof. Let $\lambda_{1}, \ldots, \lambda_{\ell}$ be all Lyapunov weights for $\alpha$. For any two dimensional subspace the Lyapunov weights $\tilde{\lambda}_{1}, \ldots, \tilde{\lambda}_{\ell}$ for the restriction $\tilde{\alpha}$ are obtained by restricting $\lambda_{1}, \ldots, \lambda_{\ell}$ to the subspace. In general these restrictions could be (positively or negatively) proportional $\mathbb{R}{\tilde{\lambda_{i}}}_{i}=\mathbb{R}{\tilde{\lambda_{j}}}_{\text {, }}$, or some of them might be trivial $\tilde{\lambda}_{k}=0$, even though this was not true for the original Lyapunov weights. However, it is easy to choose the plane so that this never happens. We claim that with this choice $\tilde{\alpha}$ has also no local rank one factors. Suppose this is not true, then there exists a proper Lie ideal $\mathfrak{h}$ in the Lie algebra $\mathfrak{g}$ of $G$ such that all weights of $\tilde{\alpha}$ on $\mathfrak{g} / \mathfrak{h}$ lie on a single line. Suppose these weights are $\lambda_{1}^{\prime}, \ldots, \lambda_{k}^{\prime}$ for some $k \leq \ell$. (For the remaining weights the weight spaces are contained in $\mathfrak{h}$.) Then the weights of $\alpha$ on $\mathfrak{g} / \mathfrak{h}$ are $\lambda_{1}, \ldots, \lambda_{k}$, which have to lie on a single line by construction of $\tilde{\alpha}$. This contradicts the assumption that $\alpha$ has no local rank factors. 
Suppose now $\alpha$ is an $\mathbb{R}^{2}$-action without local rank one factors. The Moore normal subgroup $M \subset G$ with respect to $a=\alpha^{\mathbf{t}}$ is defined to be the smallest normal subgroup such that $\operatorname{Ad}_{a}$ on $\mathfrak{g} / \mathfrak{m}$ is semisimple and all of its eigenvalues are of absolute value one. Here $\mathfrak{m}$ is the Lie algebra of $M$. It follows that $\mathfrak{m}=\mathfrak{g}$. For otherwise, $\lambda(\mathbf{t})=0$ for all weights $\lambda$ of the adjoint action of $\alpha$ on $\mathfrak{g} / \mathfrak{m}$. However, then $\mathfrak{g} / \mathfrak{m}$ would be a local rank one factor of the $\mathbb{R}^{2}$-action $\alpha$.

Therefore, the Moore normal subgroup $M=G$. Mautner's phenomenon [16, Thm. 2.1.4] now states that every $a$-fixed vector in a unitary representation of $G$ must be fixed under $M=G$. In particular, every $a$-fixed function of $L^{2}(X, m)$ must be constant function and $a$ acts ergodically with respect to the Haar measure $m$.

2.3. Entropy and a generating partition. In this section we fix some $a \in G$ and consider left multiplication by $a$ on $X=G / \Gamma$. For a countable partition $\mathcal{P} \subset \mathcal{B}_{X}$ we let

$$
\mathcal{P}^{[k, \ell)}=\bigvee_{i=k}^{\ell-1} a^{-i} \mathcal{P} .
$$

Let $U<G$ be a subgroup. We recall that a countably generated sigma algebra $\mathcal{A}$ is said to be subordinate to the foliation into $U$ orbits (subordinate to $U$ ) with respect to $\mu$ if for a.e. $x$ there exists a relatively compact neighborhood $V_{x} \subset U$ of the identity element $e \in U$ such that

$$
[x]_{\mathcal{A}}=V_{x} x .
$$

Here $[x]_{\mathcal{A}}$ is the atom of $x$, i.e. the intersection of all elements $A$ of $\mathcal{A}$ or a countable generating algebra of $\mathcal{A}$ that contain $x$.

Any countably generated sigma algebra $\mathcal{A}$ gives us a system of conditional measures $\mu_{x}^{\mathcal{A}}$ with each such measure supported on the respective atom $[x]_{\mathcal{A}}$ and the map $x \mapsto \mu_{x}^{\mathcal{A}}$ is $\mathcal{A}$ measurable. For any two countably generated sigma algebras $\mathcal{A}, \mathcal{A}^{\prime}$ so that every atom of $\mathcal{A}$ intersects at most countably many atoms of $\mathcal{A}^{\prime}$ we set

$$
\begin{aligned}
I_{\mu}\left(\mathcal{A}^{\prime} \mid \mathcal{A}\right)(x) & =-\log \mu_{x}^{\mathcal{A}}\left([x]_{\mathcal{A}^{\prime}}\right) \text { and } \\
H_{\mu}\left(\mathcal{A}^{\prime} \mid \mathcal{A}\right) & =\int I_{\mu}\left(\mathcal{A}^{\prime} \mid \mathcal{A}\right)(x) d \mu(x) .
\end{aligned}
$$

We now quote the following from [23, Prop. 9.2] (and some additional facts appearing in the proof of that proposition). These results are an adaptation of results of Ledrappier and Young [18, 19] for $C^{2}$ diffeomorphisms on compact manifolds. 
Proposition 2.2. Let $G$ be a connected Lie group, and let $\Gamma \subset G$ be a lattice in $G$. Let $a \in G$ and consider the left multiplication by a on $X=G / \Gamma$. Let

$$
U^{-}=\left\{g \in G: \lim _{n \rightarrow \infty} a^{n} g a^{-n}=e\right\}
$$

be the stable horospherical subgroup defined by $a$. Let $\mu$ be an $a$-invariant and ergodic probability measure on $X$. Then there exists a countable partition $\mathcal{P}$ of $X$ that satisfies the following properties:

(1) $\mathcal{P}$ has finite entropy $\mathrm{H}_{\mu}(\mathcal{P})<\infty$.

(2) $\mathcal{A}=\mathcal{P}^{[0, \infty)}$ is subordinate to $U^{-}$.

(3) $\mathcal{P}$ is a generating partition with respect to a, i.e. $\mathcal{P}^{(-\infty, \infty)}=\mathcal{B}_{X}$ modulo $\mu$-null sets. In particular,

$$
\mathrm{h}_{\mu}(a)=\mathrm{H}_{\mu}(a \mathcal{P} \mid \mathcal{A}) .
$$

Corollary 2.3. With the same notation as in Proposition 2.2, let $U \leq$ $U^{-}$be normalized by a. Then there exists a $\sigma$-algebra $\mathcal{A}_{U} \supset \mathcal{A}$ that satisfies

(4) $\mathcal{A}_{U}$ is subordinate to $U$.

(5) $a \mathcal{A}_{U}=a \mathcal{P} \vee \mathcal{A}_{U}$ modulo $\mu$-null sets.

(6) The entropy contribution of $U$

$$
h_{\mu}(a, U)=\mathrm{H}\left(a \mathcal{P} \mid \mathcal{A}_{U}\right)
$$

is at most the entropy $\mathrm{h}_{\mu}(a)$.

Note that by $(3), h_{\mu}(a)=h_{\mu}\left(a, U^{-}\right)$.

Before we start the proof, note that the map $u \mapsto u x$ for $u \in U^{-}$ is injective for a.e. $x$. Since $\Gamma$ is discrete, this map is clearly locally injective in the sense that for any compact subset $K \subset X$ there exists $\epsilon>0$ such that $u \mapsto u x$ is injective for $u \in B_{\epsilon}(e)$ and any fixed $x \in K$. Suppose now this map is not injective for some $x$, then there exists a non-trivial $u \in U^{-}$with $x=u x$. Then $a^{n} x=\left(a^{n} u a^{-n}\right) a^{n} x$, and since $a^{n} u a^{-n} \rightarrow e$ it follows that $a^{n} x \rightarrow \infty$ for $n \rightarrow \infty$. These points form a null set by Poincaré recurrence.

Proof of Corollary 2.3. Given a point $x_{0} \in X$ and a sufficiently small $\epsilon>0$ it is easy to define a countably generated $\sigma$-ring $\mathcal{R}$ with maximal element $B_{\epsilon}\left(x_{0}\right)$ that is subordinate to the foliation into $U$-orbits. Here the atom $[x]_{\mathcal{R}}$ will consist of $B_{2 \epsilon}^{U}(e) x \cap B_{\epsilon}\left(x_{0}\right)$ for any $x \in B_{\epsilon}\left(x_{0}\right)$.

If $x_{0} \in \operatorname{supp} \mu$, the construction in [23, Prop. 9.2] shows that one can choose $\mathcal{P}$ such that there exists an element $P_{0} \in \mathcal{P}$ with $\mu\left(P_{0}\right)>0$ and $P_{0} \subset B_{\epsilon}\left(x_{0}\right)$. Moreover, the set $V_{x} \subset U^{-}$as in (2.1) for the $\sigma$-algebra $\mathcal{A}=\mathcal{P}^{[0, \infty)}$ has diameter less than $2 \epsilon$ for all $x \in P_{0}$. We define $\mathcal{P}_{U}$ to 
be the smallest $\sigma$-algebra that contains $\mathcal{P}$ and $R \cap P_{0}$ for any $R \in \mathcal{R}$. Let

$$
\mathcal{A}_{U}=\mathcal{P}_{U}^{[0, \infty)}
$$

We claim that

$$
[x]_{\mathcal{A}_{U}}=[x]_{\mathcal{A}} \cap U x \text { for } \mu \text {-a.e. } x \text {. }
$$

Then (4) follows using the injectivity we noted before. Moreover, the claim also implies that $a \mathcal{A}_{U}$ and $a \mathcal{P} \vee \mathcal{A}_{U}$ have the same atoms for a.e. $x$, so that (5) follows as well. Then (6) follows from the definition of the entropy contribution since $\mathcal{A} \subset \mathcal{A}_{U}$.

For the claim note first that $[x]_{\mathcal{A}_{U}} \subset U x$ is obvious for $x \in P_{0}$ by the definition of $\mathcal{R}$, and follows for a.e. $x$ from that and ergodicity. Now let $y=u x \in[x]_{\mathcal{A}}$ for some $u \in U$, we need to show that $a^{n} x, a^{n} y$ belong to the same atom of $\mathcal{P}_{U}$ for all $n \geq 0$. If $a^{n} x, a^{n} y \notin P_{0}$, then this follows from the definition of $\mathcal{A}$. Otherwise, $a^{n} x$ and $a^{n} y=\left(a^{n} u a^{-n}\right)\left(a^{n} x\right)$ belong to the same $\mathcal{A}$-atom, and so $a^{n} x$ and $a^{n} y=v a^{n} x$ differ by an element $v \in V_{a^{n} x} \subset B_{2 \epsilon}^{U^{-}}(e)$ by the above mentioned property of $P_{0}$ and $\mathcal{A}$. By injectivity we must have $v=a^{n} u a^{-n} \in B_{2 \epsilon}^{U}(e)$. It follows that $a^{n} x$ and $a^{n} y$ are in the same atom of $\mathcal{R}$ which proves the claim.

2.4. Entropy contribution and conditional measures. The entropy contributions as defined in the previous subsection are dependent of the choice of sigma-algebra $\mathcal{A}_{U}$. In this section we give an alternative definition of entropy contribution which does not involve such choices. Let $a \in G$ and $U<G$ a subgroup normalized by a (later on we will assume $U$ is a subgroup of the stable horospherical subgroup $U^{-}$of a). Let $\mu$ be a probability measure on $X=G / \Gamma$ invariant under left translation by $a$.

The measure $\mu$ gives rise to a system of locally finite measures $\mu_{x, U}$, which we refer to as the conditional measures on $U$ orbits. This system of conditional measures $\mu_{x, U}$ has the following characterizing property: For any $\sigma$-algebra $\mathcal{A}_{U}$ that is subordinate to $U$ we have

$$
\mu_{x}^{\mathcal{A}_{U}}=\frac{1}{\mu_{x, U}\left(V_{x}\right)}\left(\left.\mu_{x, U}\right|_{V_{x}}\right) x \text { for a.e. } x,
$$

where $V_{x}$ is as in (2.1) and $\left(\left.\mu_{x, U}\right|_{V_{x}}\right) x$ denotes the push forward of the restriction of $\mu_{x, U}$ to $V_{x}$ under the map $u \mapsto u x$. These conditional measures are transformed in a nice way under the transformation $x \mapsto$ $a x$ : write $\theta(h)=a g a^{-1}$. Then for almost every $x$ we have that

$$
\mu_{x, U} \propto \theta_{*} \mu_{a x, U}
$$

A general construction of these conditional measures can be found in [20, Sect. 2-3]. 
We now assume $U$ is a subgroup of the stable horospherical subgroup $U^{-}$(so $\theta$, defined as above, uniformly contracts $U$ ). We define the volume decay entropy at $x$ by

$$
\operatorname{vol}_{\mu}(a, U, x)=-\lim _{n \rightarrow \infty} \frac{\log \mu_{x, U}\left(\theta^{n}\left(B_{1}^{U}(e)\right)\right)}{n}
$$

in particular, the above limits exists almost everywhere, see [4, Sect. 9.1]. This gives a pointwise definition of the entropy contribution, and we define

$$
\mathrm{h}_{\mu}(a, U)=\int \operatorname{vol}_{\mu}(a, U, x) \mathrm{d} \mu .
$$

By [4, Lemma 9.2] the two definitions (2.2) and (2.5) agree whenever the $\sigma$-algebra $\mathcal{A}_{U}$ as in Corollary 2.3 exists.

2.5. Product structure and summation formula. Using the product structure of the conditional measures, the entropy contributions of the coarse Lyapunov subgroups can be related to the total entropy. This summation formula, which we present below, is closely related to the Ledrappier-Young entropy formula [19].

Let $\mu$ be an $\alpha$-invariant and ergodic measure on $X$. Let $U^{-}$be the stable horospherical subgroup for some fixed $\alpha^{\mathbf{t}}$, and let $U^{\Lambda_{1}}, \ldots, U^{\Lambda_{\ell}} \leq$ $U^{-}$be all coarse Lyapunov subgroups contained in $U^{-}$. Then $U^{-}=$ $U^{\Lambda_{1}} \cdots U^{\Lambda_{\ell}}$ and the map that sends $\left(u_{1}, \ldots, u_{\ell}\right) \in U^{\Lambda_{1}} \times \cdots \times U^{\Lambda_{\ell}}$ to $u_{1} \cdots u_{\ell}$ is a homeomorphism (but in general not a group isomorphism). Using this homeomorphism implicitly we have

$$
\mu_{x, U^{-}} \propto \mu_{x, U^{\Lambda_{1}}} \times \cdots \times \mu_{x, U^{\Lambda_{\ell}}} \text { for a.e. } x
$$

by [4, Thm. 8.4], where $\propto$ denotes proportionality up to a multiplicative constant. This readily implies that

$$
\mathrm{h}_{\mu}\left(\alpha^{\mathbf{t}}\right)=\operatorname{vol}_{\mu}\left(\alpha^{\mathbf{t}}, U^{-}, x\right)=\sum_{i=1}^{\ell} \operatorname{vol}_{\mu}\left(\alpha^{\mathrm{t}}, U^{\Lambda_{i}}, x\right) .
$$

More generally, the above holds for any $U \leq U^{-}$that is normalized by $\alpha$, i.e.

$$
\operatorname{vol}_{\mu}\left(\alpha^{\mathbf{t}}, U, x\right)=\sum_{i=1}^{\ell} \operatorname{vol}_{\mu}\left(\alpha^{\mathbf{t}}, U \cap U^{\Lambda_{i}}, x\right) \text { a.e. }
$$

\section{An ENTROPy INEQUALity AND THE SUPPORT OF THE CONDITIONAL MEASURE}

We start our study of joinings, and will show in this section that the conditional measures for a joining have large support. Let $G=G_{1} \times G_{2}$ with Lie algebra $\mathfrak{g}=\mathfrak{g}_{1} \times \mathfrak{g}_{2}$, let $\Gamma_{i}$ be a lattice in $G_{i}$ and let $X_{i}=G_{i} / \Gamma_{i}$ 
for $i=1,2$. Define $X=X_{1} \times X_{2}$ and $\pi_{i}$ to be the projection map from $X$ to $X_{i}$ or the projection map from $G$ to $G_{i}$ for $i=1,2$ depending on the context. We will not assume that $\alpha$ has no local rank one factors.

Proposition 3.1. Let $a=\left(a_{1}, a_{2}\right) \in G_{1} \times G_{2}$, and consider left multiplication by a on $X=X_{1} \times X_{2}$ where $X_{i}=G_{i} / \Gamma_{i}$ for some lattice $\Gamma_{i}$ and $i=1,2$. Suppose $a_{i}$ acts ergodically on $X_{i}$ with respect to the Haar measure $m_{i}$ for $i=1,2$. Let $U_{1}<G_{1}$ and $U_{2}<G_{2}$ be connected unipotent subgroups, and suppose $U=U_{1} U_{2}$ is normalized by a and is contained in the stable horospherical subgroup $U^{-}$. Let $\mu$ be a (not necessarily ergodic) joining between $a_{1}$ and $a_{2}$, i.e. an a-invariant measure that projects $\left(\pi_{i}\right)_{*} \mu=m_{i}$ to the Haar measure on $X_{i}$ for $i=1,2$. Then

$$
h_{\mu}(a, U) \leq h_{m_{1}}\left(a_{1}, U_{1}\right)+h_{\mu}\left(a, U_{2}\right)
$$

Proof. We will need the following partitions and $\sigma$-algebras. For $i=$ 1,2 we use the ergodic action of $a_{i}$ on $X_{i}$ and Proposition 2.2 to find generating countable partitions $\overline{\mathcal{P}}_{i}$ of $X_{i}$ with finite $m_{i}$-entropy so that $\overline{\mathcal{P}}_{i}^{[0, \infty)}$ is subordinate to the stable horospherical subgroup $U_{i}^{-}<G_{i}$ of $a_{i}$. Let $\mathcal{P}_{i}=\pi_{i}^{-1}\left(\overline{\mathcal{P}}_{i}\right)$ be the corresponding partition of $X$, and set $\mathcal{P}=\mathcal{P}_{1} \vee \mathcal{P}_{2}$. Since $\mu$ is a joining, it follows that $\mathcal{P}$ has finite $\mu$-entropy and is generating for $a$. Moreover, $\mathcal{P}^{[0, \infty)}$ is subordinate to the stable horospherical subgroup $U^{-}<G$ of $a$. Now apply Corollary 2.3 to find for $i=1,2$ the $\sigma$-algebra $\overline{\mathcal{A}}_{U_{i}} \subset \mathcal{B}_{X_{i}}$ that is subordinate to $U_{i}$. Define $\mathcal{A}_{i}=\pi_{i}^{-1} \overline{\mathcal{A}}_{U_{i}}$ for $i=1,2$ and $\mathcal{A}_{U}=\mathcal{A}_{1} \vee \mathcal{A}_{2}=\overline{\mathcal{A}}_{U_{1}} \times \overline{\mathcal{A}}_{U_{2}}$. As above, it follows that $\mathcal{A}_{U}$ is subordinate to $U$ and that $\mathcal{B}_{X_{1}} \times \overline{\mathcal{A}}_{U_{2}}$ is subordinate to $U_{2}$ since $\mu$ is a joining.

The various entropy contributions are now defined by

$$
\begin{aligned}
\mathrm{h}_{\mu}(a, U) & =\mathrm{H}_{\mu}\left(a \mathcal{P} \mid \mathcal{A}_{U}\right), \\
\mathrm{h}_{m_{1}}\left(a_{1}, U_{1}\right) & =\mathrm{H}_{m_{1}}\left(a_{1} \overline{\mathcal{P}}_{1} \mid \overline{\mathcal{A}}_{U_{1}}\right), \text { and } \\
\mathrm{h}_{\mu}\left(a, U_{2}\right) & =\mathrm{H}_{\mu}\left(a \mathcal{P} \mid \mathcal{B}_{X_{1}} \times \overline{\mathcal{A}}_{U_{2}}\right) .
\end{aligned}
$$

Applying Corollary 2.3 (5) for $\overline{\mathcal{A}}_{U_{i}}$ and $i=1,2$, we see that $a \mathcal{P} \vee$ $\mathcal{A}_{U}=a \mathcal{A}_{U}$. Therefore, we get

$$
\begin{aligned}
\mathrm{h}_{\mu}(a, U) & =\frac{1}{n} \mathrm{H}_{\mu}\left(a^{n} \mathcal{A}_{U} \mid \mathcal{A}_{U}\right)=\frac{1}{n} \mathrm{H}_{\mu}\left(\mathcal{P}^{[-n, 0)} \mid \mathcal{A}_{U}\right) \\
& =\frac{1}{n} \mathrm{H}_{\mu}\left(\mathcal{P}_{1}^{[-n, 0)} \mid \mathcal{A}_{U}\right)+\frac{1}{n} \mathrm{H}_{\mu}\left(\mathcal{P}_{2}^{[-n, 0)} \mid \mathcal{A}_{U} \vee \mathcal{P}_{1}^{[-n, 0)}\right)
\end{aligned}
$$


We first deal with the first term on the right hand side of (3.4). Since $\mathcal{A}_{1}=\pi_{1}^{-1} \overline{\mathcal{A}}_{U_{1}} \subset \mathcal{A}_{U}$, by the properties of conditional entropy and (3.2),

$$
\begin{aligned}
\frac{1}{n} \mathrm{H}_{\mu}\left(\mathcal{P}_{1}^{[-n, 0)} \mid \mathcal{A}_{U}\right) & \leq \frac{1}{n} \mathrm{H}_{\mu}\left(\mathcal{P}_{1}^{[-n, 0)} \mid \mathcal{A}_{1}\right) \\
& =\frac{1}{n} \mathrm{H}_{m_{1}}\left(\overline{\mathcal{P}}_{1}^{[-n, 0)} \mid \overline{\mathcal{A}}_{U_{1}}\right)=\mathrm{h}_{m_{1}}\left(a_{1}, U_{1}\right) .
\end{aligned}
$$

We now proceed to consider the second term on the right hand side of (3.4):

$$
\begin{aligned}
\frac{1}{n} \mathrm{H}_{\mu}\left(\mathcal{P}_{2}^{[-n, 0)} \mid \mathcal{A}_{U} \vee \mathcal{P}_{1}^{[-n, 0)}\right) & =\frac{1}{n} \sum_{k=1}^{n} \mathrm{H}_{\mu}\left(a^{k} \mathcal{P}_{2} \mid \mathcal{A}_{U} \vee \mathcal{P}_{1}^{[-n, 0)} \vee \mathcal{P}_{2}^{[-k+1,0)}\right) \\
& =\frac{1}{n} \sum_{k=1}^{n} \mathrm{H}_{\mu}\left(a^{k} \mathcal{P}_{2} \mid a^{k-1} \mathcal{A}_{U} \vee \mathcal{P}_{1}^{[-n,-k)}\right) \\
& =\frac{1}{n} \sum_{k=1}^{n} \mathrm{H}_{\mu}\left(\mathcal{P}_{2} \mid \mathcal{A}_{U} \vee \mathcal{P}_{1}^{[-n+k, 0)}\right)
\end{aligned}
$$

and by the martingale convergence theorem [26, Ch. 2, Thm. 6] for entropy one sees that

$$
\mathrm{H}_{\mu}\left(\mathcal{P}_{2} \mid \mathcal{A}_{U} \vee \mathcal{P}_{1}^{[-n+k, 0)}\right) \rightarrow \mathrm{H}_{\mu}\left(\mathcal{P}_{2} \mid \mathcal{A}_{U} \vee \mathcal{P}_{1}^{(-\infty, 0)}\right)
$$

However, by definition $\mathcal{A}_{U}$ contains $\mathcal{P}_{1}^{[0, \infty)}$, and since $\overline{\mathcal{P}}_{1}$ is generating for $a_{1}$ we see that $\mathcal{A}_{U} \vee \mathcal{P}_{1}^{(-\infty, 0)}=\mathcal{B}_{X_{1}} \times \overline{\mathcal{A}}_{U_{2}}$. From (3.3) it follows that

$$
\mathrm{H}_{\mu}\left(\mathcal{P}_{2} \mid \mathcal{A}_{U} \vee \mathcal{P}_{1}^{(-\infty, 0)}\right)=\mathrm{h}_{\mu}\left(T, U_{2}\right)
$$

and the average in (3.6) converges to $h_{\mu}\left(a, U_{2}\right)$ which establishes (3.1).

Proposition 3.2. Let $a, X, U, \mu$ be as in Proposition 3.1. If equality holds in (3.1) then $U_{1}$ is the smallest connected subgroup that is normalized by $a$ and contains $\pi_{1}\left(\operatorname{supp} \mu_{x, U}\right)$ for a.e. $x$.

Proof. Suppose by contradiction that $U_{1}^{\prime}<U_{1}$ is a proper connected subgroup that contains $\pi_{1}\left(\operatorname{supp} \mu_{x, U}\right)$ for a.e. $x$ and is normalized by $a_{1}$. Then the conditional measure $\mu_{x, U}$ is supported by $U^{\prime}=U_{1}^{\prime} U_{2}$ and so satisfies $\left.\left(\mu_{x, U}\right)\right|_{U^{\prime}}=\mu_{x, U^{\prime}}$ for a.e. $x$. It follows from the pointwise definition of the entropy contribution in (2.4) and Proposition 3.1 that

$$
\begin{aligned}
h_{\mu}(a, U)=h_{\mu}\left(a, U^{\prime}\right) \leq \mathrm{h}_{m_{1}}\left(a_{1}, U_{1}^{\prime}\right)+h_{\mu}\left(a, U_{2}\right) & \\
& <h_{m_{1}}\left(a_{1}, U_{1}\right)+h_{\mu}\left(a, U_{2}\right),
\end{aligned}
$$


where the last strict inequality follows easily from the fact that $U_{1}^{\prime} \subset U_{1}$ is a proper subgroup and $a$ uniformly contracts $U_{1}$. This is a contradiction to our assumption.

Proposition 3.3. Let $a, \mu$, and $X=X_{1} \times X_{2}$ be as in Proposition 3.1. For the stable horospherical subgroup $U^{-}=U_{1}^{-} U_{2}^{-}$of a we have equality in (3.1).

Proof. The Abramov-Rohklin conditional entropy formula gives that

$$
\mathrm{h}_{\mu}(a)=\mathrm{h}_{m_{1}}\left(a_{1}\right)+\mathrm{h}_{\mu}\left(a \mid \pi_{1}^{-1} \mathcal{B}_{X_{1}}\right) \text {. }
$$

By Proposition 2.2 we have $h_{\mu}\left(a, U^{-}\right)=h_{\mu}(a)$ and $h_{m_{1}}\left(a_{1}, U_{1}^{-}\right)=$ $h_{m_{1}}\left(a_{1}\right)$. It remains to match the last term of the above and (3.1). By (3.3)

$$
\mathrm{h}_{\mu}\left(a, U_{2}^{-}\right)=\mathrm{H}_{\mu}\left(a \mathcal{P} \mid \mathcal{B}_{X_{1}} \times \overline{\mathcal{A}}_{U_{2}^{-}}\right)=\mathrm{H}_{\mu}\left(a \mathcal{P} \mid \mathcal{B}_{X_{1}} \times \overline{\mathcal{P}}_{2}^{[0, \infty]}\right),
$$

where we used that $\overline{\mathcal{P}}_{2}^{[0, \infty)}$ is $U_{2}^{-}$-foliated by Proposition 2.2. However, the last expression equals the conditional entropy $\mathrm{h}_{\mu}\left(a \mid \pi_{1}^{-1} \mathcal{B}_{X_{1}}\right)$ since $\mathcal{P}$ is a generating partition for $a$.

We can now use the product structure to deduce a similar result regarding the coarse Lyapunov foliations.

Corollary 3.4. Let $\alpha: \mathbb{R} \rightarrow G$ be an Ad-semisimple homomorphism such that for every nontrivial $\mathbf{t}$ the transformation $\alpha_{i}^{\mathbf{t}}$ on $X_{i}$ acts ergodically with respect to $m_{i}$ for $i=1,2$. Let $\mu$ be a joining between $\alpha_{1}$ and $\alpha_{2}$. Then we also have equality in (3.1) for a coarse Lyapunov subgroup $U=U^{\Lambda}=U_{1}^{\Lambda} U_{2}^{\Lambda} \leq U^{-}$.

Proof. Let $\mu$ be $\alpha$-invariant. Let $U^{\Lambda}$ be a coarse Lyapunov subgroup. Choose some $a=\alpha^{\mathbf{t}}$ such that $U^{\Lambda}$ is contained in the stable horospherical subgroup $U^{-}$to $a$. Let $U^{-}=U^{\Lambda_{1}} \cdots U^{\Lambda_{\ell}}$ be the decomposition of the stable horospherical subgroup into coarse Lyapunov subgroups as in Section 2.5 with $\Lambda=\Lambda_{1}$. For any $j$ Proposition 3.1 gives

$$
h_{\mu}\left(a, U^{\Lambda_{j}}\right) \leq h_{m_{1}}\left(a_{1}, U_{1}^{\Lambda_{j}}\right)+h_{\mu}\left(a, U_{2}^{\Lambda_{j}}\right) .
$$

Taking the sum over $j$, each term here sums up the corresponding term for $U^{-}=U_{1}^{-} U_{2}^{-}$by (2.7)-(2.8). However, we already showed that the latter satisfy an exact summation formula so the same has to be true for the coarse Lyapunov subgroups.

The following is now a direct consequence of Proposition 3.2 and Corollary 3.4: 
Corollary 3.5. Let $U=U_{1}^{\Lambda} U_{2}^{\Lambda}$ be a coarse Lyapunov subgroup for $\alpha$, and let $P \leq U$ be the smallest connected subgroup that is normalized by $\alpha$ such that $\operatorname{supp} \mu_{x, U} \subset P$ for a.e. $x$. Then $\pi_{i}(P)=U_{i}^{\Lambda}$ for $i=1,2$.

In the notations of Corollary 3.5, for every $x \in X$ let $P_{x} \leq U$ denote the smallest connected subgroup normalized by $\alpha$ containing supp $\mu_{x, U}$. Since a connected Lie group is determined by its Lie algebra, we can give the set of subgroups of $U$ a natural topology coming from the Grassmannians of all dimensions of Lie $U$. The map $x \mapsto P_{x}$ is measurable (we leave the straightforward but tedious verification of this fact to the pedantic reader) and is (modulo null sets) $\alpha$ invariant, hence if $\mu$ is $\alpha$ ergodic $P_{x}$ is constant almost everywhere. This constant Lie group is necessarily the group $P$ of Corollary 3.5 and so we obtain

Corollary 3.6. Let $\mu$ be an $\alpha$-ergodic joining of $X_{1}$ and $X_{2}$ as above, and Let $U=U_{1}^{\Lambda} U_{2}^{\Lambda}$ be a coarse Lyapunov subgroup for $\alpha$. For every $x$, let $P_{x}$ denote the smallest connected subgroup $P_{x} \leq U$ that is normalized by $\alpha$ containing $\operatorname{supp} \mu_{x, U}$. Then there is a group $P \leq U$ with $\pi_{i}(P)=$ $U_{i}^{\Lambda}$ for $i=1,2$, so that for a.e. $x$ we have that $P_{x}=P$.

\section{The High ENTROPy THEOREM}

As mentioned before, our main tool is the high entropy case as in [4, Thm. 8.5]. We will need the following special case.

Theorem 4.1. Let $G$ be a Lie group, let $\Gamma \subset G$ be a discrete subgroup, and let $X=G / \Gamma$. Let $\alpha: \mathbb{R}^{n} \rightarrow G$ be an $\mathbb{R}$-diagonalizable homomorphism, and let $U^{-}$be the stable horospherical subgroup to some element $a=\alpha^{\mathbf{t}}$. Furthermore, let $U^{-}=U^{\Lambda_{1}} \cdots U^{\Lambda_{\ell}}$ be the decomposition of $U^{-}$ into coarse Lyapunov subgroups. For any $\alpha$-invariant probability measure $\mu$ there exist for a.e. $x$ two subgroups

$$
H_{x} \subseteq P_{x} \subseteq U^{-}
$$

with the following properties:

(1) $\mu_{x, U^{-}}$is supported by $P_{x}$.

(2) $\mu_{x, U^{-}}$is left- and right-invariant under multiplication with elements of $H_{x}$.

(3) $H_{x}$ and $P_{x}$ are connected and their Lie algebras are direct sums of subspaces of the weight spaces.

(4) $H_{x}$ is a normal subgroup of $P_{x}$ and any elements $g \in P_{x} \cap U^{\Lambda_{r}}$ and $h \in P_{x} \cap U^{\Lambda_{s}}$ of different coarse Lyapunov subgroups $(r \neq s)$ satisfy that $g H_{x}$ and $h H_{x}$ commute with each other in $P_{x} / H_{x}$.

(5) $\mu_{x, U^{\Lambda_{i}}}$ is left-and right-invariant under multiplication with elements of $H_{x} \cap U^{\Lambda_{i}}$ for $i=1, \ldots, \ell$. 
We quote also (a special case of) [4, Lemma 9.6] and its short proof since it is crucial to this paper.

Lemma 4.2. Let $G$ be a connected Lie group, let $\Gamma \subset G$ be a lattice, and let $\alpha: \mathbb{R}^{n} \rightarrow G$ be a homomorphism. Let $\mathfrak{g}$ be the Lie algebra of $G$, and let $\mathfrak{g}^{\zeta}$ denote the weight space associated for any weight $\zeta$. Let $V$ be a subspace of the dual of $\mathbb{R}^{n}$. Then $\mathfrak{g}^{(V)}=\left\langle\mathfrak{g}^{\zeta},\left[\mathfrak{g}^{\zeta}, \mathfrak{g}^{\xi}\right]: \zeta, \xi \notin V\right\rangle$ is a Lie ideal in $\mathfrak{g}$.

Therefore, if there are no rank one factors, then every element of $\mathfrak{g}^{\lambda}$ is a sum of expressions $[v, w]$ with $v \in \mathfrak{g}^{\zeta}, w \in \mathfrak{g}^{\xi}$ and $\zeta, \xi \notin \mathbb{R} \lambda$.

Proof. Since $\operatorname{Ad}_{a}\left(\mathfrak{g}^{\zeta}\right)=\mathfrak{g}^{\zeta}$ and $\operatorname{Ad}_{a}([u, v])=\left[\operatorname{Ad}_{a}(u), \operatorname{Ad}_{a}(v)\right]$ for any $a=\alpha^{\mathbf{t}}$, it follows that $\mathfrak{g}^{(V)}$ is invariant under the adjoint action induced by $\alpha$. Therefore, $\mathfrak{g}^{(V)}$ splits into a sum of subspaces of the weight spaces. We need to show that $\left[\mathfrak{g}^{\eta}, \mathfrak{g}^{(V)}\right] \subseteq \mathfrak{g}^{(V)}$ for all $\eta$ (including $\eta=0$ ).

Suppose first that $u \in \mathfrak{g}^{\eta}$ and $v \in \mathfrak{g}^{\zeta} \cap \mathfrak{g}^{(V)}$ with $\zeta \notin V$. If $\eta \notin V$ then $[u, v] \in \mathfrak{g}^{(V)}$ by definition of $\mathfrak{g}^{(V)}$, and otherwise $[u, v] \in \mathfrak{g}^{\eta+\zeta} \subseteq \mathfrak{g}^{(V)}$ because $\eta+\zeta \notin V$ and again the definition.

Let $u \in \mathfrak{g}^{\eta}, v \in \mathfrak{g}^{\zeta}$, and $w \in \mathfrak{g}^{\xi}$ with $\zeta, \xi \notin V$ (so that $[v, w] \in \mathfrak{g}^{(V)}$ ). Again, if $\eta+\zeta+\xi \notin V$ then there is nothing to show. So assume

$\eta+\zeta+\xi \in V$. If $\eta \notin V$ then $\zeta+\xi \notin V$ and we are again done. The remaining case is $\eta, \eta+\zeta+\xi \in V$. By the Jacobi identity $[u,[v, w]]=$ $-[v,[w, u]]-[w,[u, v]]$ and the two expressions on the right belong to $\mathfrak{g}^{(V)}$ since $\zeta, \xi+\eta, \xi, \eta+\zeta \notin V$.

For the final statement let $V=\mathbb{R} \lambda$. Then $\mathfrak{g}^{(V)}$ defines a Lie ideal such that the adjoint action on $\mathfrak{g} / \mathfrak{g}^{(V)}$ has no weights outside of $V$. Since there are no rank one factors, we have $\mathfrak{g}^{(V)}=\mathfrak{g}$ and every $u \in \mathfrak{g}^{\lambda}$ belongs to $\mathfrak{g}^{(V)}$. By restricting the sum that expresses $u$ to those terms that belong to $\mathfrak{g}^{\lambda}$ the lemma follows.

Note that in particular since $\mathfrak{g}^{\eta}$ is nilpotent for every $\eta \neq 0$, the above lemma implies that a connected Lie group with no local rank one factors is generated by unipotent one parameter subgroups.

\section{Translation invariance of the CONDItional MEASURES AND THE JOINING}

In this section we will use the assumption that the adjoint action to $\alpha$ has real eigenvalues and that there are no local rank one factors by applying the main results of [4]. For $g \in G$ write $L_{g}(h)=g h$ for the left action of $g$ on $G$. We simply say a measure is $g$-invariant if it is invariant under $L_{g}$.

If $\mu$ is an ergodic joining between $\alpha_{1}$ and $\alpha_{2}$ as in Theorem 1.4, then by Proposition 2.1 (applied to $m_{1} \times m_{2}$ ) it contains a subaction $\tilde{\alpha}$ with 
the same coarse Lyapunov subgroups such that any element of $\tilde{\alpha}_{i}$ acts ergodically on $X_{i}$ with respect to $m_{i}$ for $i=1,2$. So, the results of Section 3 apply to this situation.

Proposition 5.1. For every coarse Lyapunov subgroup $U^{\Lambda}=U_{1}^{\Lambda} U_{2}^{\Lambda}$ the connected subgroup

$$
I^{\Lambda}=\left\{u \in U^{\Lambda}: \mu \text { is u-invariant }\right\}
$$

satisfies $\pi_{i}\left(\mathfrak{i}^{\Lambda}\right)=\mathfrak{u}_{i}^{\Lambda}$ for $i=1,2$. Here $\mathfrak{i}^{\Lambda}$ is the Lie algebra of $I^{\Lambda}$ and $\mathfrak{u}_{i}^{\Lambda}$ is the Lie algebra of $U_{i}^{\Lambda}$.

Proof. It is easy to see that $I^{\Lambda}$ is closed and normalized by $\alpha$ which implies that $I^{\Lambda}$ is also connected. Similarly it has been shown in [4, Prop. 6.2] for a.e. $x$ that

$$
I_{x}^{\Lambda}=\left\{u \in U^{\Lambda}: \mu_{x, U^{\Lambda}} \text { is } u \text {-invariant }\right\}
$$

is connected and that its Lie algebra is a direct sum of subspaces of $\mathfrak{u}^{\lambda}$ for $\lambda \in \Lambda$. Since we assume that the eigenvalues of the adjoint representation of $\alpha$ are real, we conclude that the Lie algebra of $I_{x}^{\Lambda}$ is a direct sum of eigenspaces. Therefore, $I_{x}^{\Lambda}$ is normalized by $\alpha$ which shows together with (2.3) that $I_{x}^{\Lambda}=I_{\alpha^{\mathbf{t}} x}^{\Lambda}$ for every $\mathbf{t} \in \mathbb{R}^{n}$ and a.e. $x \in X$.

The map $x \mapsto I_{x}^{\Lambda}$ is measurable (we again omit the details which are straightforward but tedious) and so by ergodicity we conclude that $I_{x}^{\Lambda}$ is independent of $x$ a.e., and by [20, Prop. 4.3] we conclude that $I_{x}^{\Lambda}=I^{\Lambda}$ for a.e. $x$.

Let $\lambda \in \Lambda$ and $v \in \mathfrak{u}_{1}^{\lambda}$. Applying Lemma 4.2 (which makes use of the assumption that there are no local rank one factors) we see that $v$

can be expressed as a finite sum of terms $\left[w_{1}, w_{2}\right]$ with $w_{1} \in \mathfrak{u}_{1}^{\lambda_{1}}$, $w_{2} \in \mathfrak{u}_{1}^{\lambda_{2}}$, and $\lambda_{1}, \lambda_{2}$ linearly independent. We will show that the all these terms $\left[w_{1}, w_{2}\right]$ belongs to the Lie algebra $\pi_{1}\left(\mathfrak{i}^{\Lambda}\right)$. Then $v \in \pi_{1}\left(\mathfrak{i}^{\Lambda}\right)$ and the proposition will follow.

So consider now $w_{1} \in \mathfrak{u}_{1}^{\lambda_{1}}, w_{2} \in \mathfrak{u}_{1}^{\lambda_{2}}$, and $\lambda_{1}, \lambda_{2}$ linearly independent. Since $\lambda_{i}$ are the weights, $\left[w_{1}, w_{2}\right] \in \mathfrak{u}_{1}^{\lambda}$ and $\lambda=\lambda_{1}+\lambda_{2}$. By the linear independence there exists some $\mathbf{t} \in \mathbb{R}^{n}$ such that $\lambda(\mathbf{t}), \lambda_{1}(\mathbf{t}), \lambda_{2}(\mathbf{t})<0$, in other words the stable horospherical subgroup $U^{-}$corresponding to $a=\alpha^{\mathbf{t}}$ contains $U^{\Lambda}, U^{\Lambda_{1}}$, and $U^{\Lambda_{2}}$ (where $\Lambda_{j}=\mathbb{R}^{+} \lambda_{j}$ ).

Next we apply Theorem 4.1 to $U^{-}$: for a.e. $x$ there exist two closed connected subgroups $H_{x} \leq P_{x} \leq U^{-}$such that $\mu_{x, U^{-}}$is supported by $P_{x}$ and is invariant under elements of $H_{x}$, and $g_{j} \in P_{x} \cap U^{\Lambda_{j}}$ for $j=1,2$ implies $\left[g_{1}, g_{2}\right] \in H_{x}$. Furthermore, $H_{x}$ and $P_{x}$ are normalized by $\alpha$, since their Lie algebras are direct sums of subspaces of $\mathfrak{u}^{\zeta}$ for various $\zeta$ and since we assume that $\alpha$ is $\mathbb{R}$-diagonalizable. By Corollary 3.6 there 
exists a connected subgroup $P \leq U^{-}$that is normalized by $\alpha$ such that supp $\mu_{x, U} \subset P \leq P_{x}$ for a.e. $x \in X$.

Corollary 3.5 implies now that $\pi_{1}\left(P_{x}\right)=U_{1}$ for a.e. $x$. Therefore, there exist for $j=1,2$ elements $g_{j} \in P_{x}$ such that $\pi_{1}\left(g_{j}\right)=\exp \left(w_{j}\right)$. Since the Lie algebra of $P_{x}$ is a direct sum of subspaces of the $\mathfrak{u}^{\zeta}$, we can assume here that $g_{j} \in P_{x} \cap U^{\Lambda_{j}}$. However, then $\left[g_{1}, g_{2}\right] \in H_{x}$ by Theorem 4.1. Note that $\pi_{1}\left(\left[g_{1}, g_{2}\right]\right)=\exp \left(\left[w_{1}, w_{2}\right]+\ldots\right)$ by the Campbell-Hausdorff formula,

where the dots indicate elements of $\mathfrak{u}^{\zeta}$ for various $\zeta \neq \lambda$. Therefore and since the Lie algebra of $H_{x}$ is a direct sum of subspaces of Lyapunov spaces, we conclude that $H_{x}$ contains an element $g \in U^{\Lambda}$ with $\pi_{1}(g)=\exp \left(\left[w_{1}, w_{2}\right]\right)$. By the properties of $H_{x}$ we know that $\mu_{x, U^{-}}$is left invariant under $g$. By (2.6) we know $\mu_{x, U^{-}}$is a product measure, where we can assume that $\Lambda_{1}=\Lambda$ is the first coarse Lyapunov weight that appears. However, this means that $\mu_{x, U^{\Lambda}}$ is left invariant under $g$ and so $g \in I_{x}^{\Lambda}=I^{\Lambda}$. Since $I^{\Lambda}$ is connected, we have $\log g \in \mathfrak{i}^{\Lambda}$ and so $\left[w_{1}, w_{2}\right] \in \pi_{1}\left(\mathfrak{i}^{\Lambda}\right)$ as claimed.

Let $I$ be the subgroup generated by all Ad-unipotent one-parameter subgroups that preserve $\mu$, and let $\mathfrak{i}$ be its Lie algebra.

Corollary 5.2. $\pi_{i}(\mathfrak{i})=\mathfrak{g}_{i}$ for $i=1,2$. Moreover, $\mathfrak{i} \cap\left(\{0\} \times \mathfrak{g}_{2}\right)$ is a Lie ideal.

Proof. By Lemma 4.2 the Lie algebra $\mathfrak{h}_{i}$ generated by $\mathfrak{u}_{i}^{\zeta}$ for non-zero $\zeta$ is a Lie ideal in $\mathfrak{g}_{i}$, so that $\mathfrak{h}_{i}=\mathfrak{g}_{i}$ by the assumption that there are no local rank one factors for $\alpha_{i}$. The first statement now follows from Proposition 5.1

For the second statement, suppose $(0, w) \in \mathfrak{i}$ and $\left(v_{1}, v_{2}\right) \in \mathfrak{g}$. Then there exists some $v_{1}^{\prime}$ with $\left(v_{1}^{\prime}, v_{2}\right) \in \mathfrak{i}$, and so

$$
\left[(0, w),\left(v_{1}, v_{2}\right)\right]=\left[(0, w),\left(v_{1}^{\prime}, v_{2}\right)\right]=\left(0,\left[w, v_{2}\right]\right) \in \mathfrak{i}
$$

Note that $I$ is a subgroup for which Ratner's theorem [28] applies. So it remains to show that $\mu$ is an $I$-invariant and ergodic measure, which will imply that $\mu$ is algebraic.

Let $J \leq G$ be the group generated by all one-parameter subgroups that preserve $\mu$ and let $\mathfrak{j}$ be its Lie algebra. Clearly $\alpha\left(\mathbb{R}^{n}\right) I \subset J$ so that $\mathfrak{a}+\mathfrak{i} \subset \mathfrak{j}$ where $\mathfrak{a}$ is the Lie algebra of $\alpha\left(\mathbb{R}^{n}\right)$.

Further, denote by $\mathfrak{n} \subset \mathfrak{r}$ the nil radical and the radical of $\mathfrak{g}$, i.e. $\mathfrak{n}$ is the maximal nilpotent Lie ideal and $\mathfrak{r}$ is the maximal solvable ideal.

Lemma 5.3. We have $\mathfrak{j} \cap \mathfrak{n} \subset \mathfrak{i}$. 
Proof. Let $v \in \mathfrak{j} \cap \mathfrak{n}$. Then $\mu$ is invariant under $\exp (t v)$ for $t \in \mathbb{R}$ by definition of $\mathfrak{j}$, and $\operatorname{ad}_{v}$ is a nilpotent endomorphism of $\mathfrak{g}$ since $\mathfrak{n}$ is a nilpotent ideal. It follows that $\exp (t v)$ is Ad-unipotent, and so $v \in \mathfrak{i}$ by definition.

Lemma 5.4. If $\alpha$ has no local rank one factors, then $\mathfrak{n}=\mathfrak{r}$.

Proof. By [33, Thm. 3.8.3(iii)] we have $[\mathfrak{g}, \mathfrak{r}] \subset \mathfrak{n}$. Therefore, $\mathfrak{r}^{\prime}=\mathfrak{r} / \mathfrak{n}$ is in the center and simultaneously the radical of $\mathfrak{g}^{\prime}=\mathfrak{g} / \mathfrak{n}$. If $\mathfrak{g}^{\prime}=\mathfrak{g}_{s}^{\prime}+\mathfrak{r}^{\prime}$ is the Levi decomposition, then $\mathfrak{g}_{s}^{\prime}$ is an ideal. Therefore, $\mathfrak{r}^{\prime}$ is an abelian factor of $\mathfrak{g}$. By the assumption that there are no local rank one factors we must have $\mathfrak{r}^{\prime}=0$.

Proof of Theorem 1.4. It suffices to show that $\mathfrak{a} \subset \mathfrak{i}$. Because then $\alpha\left(\mathbb{R}^{n}\right) \subset I$ which means that $\mu$ is $I$-invariant and ergodic. By Ratner's theorem [28] this implies that $\mu$ is algebraic.

So let $v=\left(v_{1}, v_{2}\right) \in \mathfrak{a}$. By Corollary 5.2 it follows that there exists $v_{2}^{\prime} \in \mathfrak{g}_{2}$ such that $\left(v_{1}, v_{2}^{\prime}\right) \in \mathfrak{i}$. Then $\left(0, v_{2}-v_{2}^{\prime}\right)=(0, w)$ belongs to $\mathfrak{j}$ - the Lie algebra of the group $J$ generated by all one parameter subgroups that preserve $\mu$.

Moreover, since $I$ is normalized by $\alpha$, its Lie algebra $\mathfrak{i}$ is a direct sum of subspaces of weight spaces. Since $\left(v_{1}, v_{2}^{\prime}\right) \in \mathfrak{i}$ and $\left(v_{1}, v_{2}\right) \in \mathfrak{a} \subset \mathfrak{u}^{0}$, this shows we can choose $v_{2}^{\prime}$ so that $\left(v_{1}, v_{2}^{\prime}\right),(0, w) \in \mathfrak{u}^{0}$, i.e. $w$ is fixed under the adjoint representation restricted to $\alpha_{2}$. By the first statement of Corollary 5.2 it follows that $[(0, w), \mathfrak{i}]=\left(0,\left[w, \mathfrak{g}_{2}\right]\right) \subset \mathfrak{j}$. Since $(0, w) \in \mathfrak{u}^{0}$ we have $\left(0,\left[w, \mathfrak{u}_{2}^{\lambda}\right]\right) \subset \mathfrak{u}^{\lambda}$. For any nonzero $\lambda$ any element of $\mathfrak{u}^{\lambda}$ gives rise to a Ad-unipotent one parameter subgroup, therefore, $\left(0,\left[w, \mathfrak{u}_{2}^{\lambda}\right]\right)=i$. By our assumption that there are no local rank one factors and by Lemma 4.2 every element of $\mathfrak{u}^{0}$ can be written as a linear sum of expressions $\left[u, u^{\prime}\right]$ with $u \in \mathfrak{u}_{2}^{\lambda}$ and $u^{\prime} \in \mathfrak{u}_{2}^{-\lambda}$ for some nonzero $\lambda$. By the second statement of Corollary 5.2 the Lie algebra $\mathfrak{i}_{2}$ with $\mathfrak{i} \cap\left(\{0\} \times \mathfrak{g}_{2}\right)=\{0\} \times \mathfrak{i}_{2}$ is actually a Lie ideal in $\mathfrak{g}_{2}$. The Jacobi identity now implies that

$$
\left[w,\left[u, u^{\prime}\right]\right]=-\left[u,\left[u^{\prime}, w\right]\right]-\left[u^{\prime},[w, u]\right] \in \mathfrak{i}_{2} .
$$

Therefore, $\left[w, \mathfrak{g}_{2}\right] \subset \mathfrak{i}_{2}$.

Since $\alpha_{2}$ has no local rank one factors, it follows from Lemma 5.4 that the nil radical $\mathfrak{n}_{2}$ of $\mathfrak{g}_{2}$ is also the radical. Therefore $\overline{\mathfrak{g}_{2}}=\mathfrak{g}_{2} / \mathfrak{n}_{2}$ is semisimple and it follows that $\bar{w}=w+\mathfrak{n}_{2}$ belongs to the Lie ideal generated by $\left[\bar{w}, \overline{\mathfrak{g}_{2}}\right]$. Therefore, $w \in \mathfrak{i}_{2}+\mathfrak{n}_{2}$ and there exists some $w^{\prime} \in \mathfrak{i}_{2}$ with $w-w^{\prime} \in \mathfrak{n}_{2}$. However, since both $(0, w),\left(0, w^{\prime}\right) \in \mathfrak{j}$ the same applies to $\left(0, w-w^{\prime}\right) \in\{0\} \times \mathfrak{n}_{2}$. By Lemma $5.3\left(0, w-w^{\prime}\right) \in \mathfrak{i}$ and so $(0, w), v \in \mathfrak{i}$ as claimed. 


\section{FACTORS OF HIGHER RANK ABELIAN ACTIONS}

Let $G$ be a connected Lie group, $\Gamma$ a lattice in $G$, and $m$ Haar measure on $X=G / \Gamma$. Let $\alpha: \mathbb{R}^{n} \rightarrow G$ be an injective homeomorphism diagonalizable over $\mathbb{R}$ with no local rank one factors, and set $A=$ $\alpha\left(\mathbb{R}^{n}\right)$. For every $g \in G$ we let $L_{g}$ and $R_{g}$ denote the element of Aff $G$ given by left (respectively right) translation, i.e. $L_{g}(h)=g h$ and $R_{g}(h)=h g$ for any $h \in G$. Furthermore, if $H$ is a subgroup of $G$ we let $L_{H}=\left\{L_{h}: h \in H\right\}$ and $R_{H}=\left\{R_{h}: h \in H\right\}$. We let Aff ${ }_{1} G<$ Aff $G$ denote those affine groups which preserve a Haar measure on $G$ (since $G$ has a lattice it is in particular unimodular and so we need not distinguish between left and right Haar measures). Note that both $L_{G}$ and $R_{G}$ are normal subgroups of $\mathrm{Aff}_{1} G$.

In this section we want to classify measurable factors (also called quotients) of $G / \Gamma$, or what amounts to the same countably generated $A$-invariant sigma algebras $\mathcal{A}$ of Borel subsets of $G / \Gamma$. Our input to this classification of factors is the classification of self joining; we will also use the fact that $G$ is generated by unipotent one parameter subgroups (which follows from the local rank assumption) though this is a minor technical point. Essentially everything we do in this section is contained in Dave Witte Morris papers [34, 35] and the references cited there, in particular [3]; we have tried to give here a slightly more streamlined version of his proof (on the other hand, Witte Morris' setup, particularly in [35] is more general than ours).

It would be more convenient for us to view $\mathcal{A}$ as a left $A$-invariant sigma algebra of right $\Gamma$-invariant subsets of $G$ (notice the asymmetric role of $A$ and $\Gamma$ : for every set $B \in \mathcal{A}$ and $\gamma \in \Gamma$ we have that $R_{\gamma} B=B$ whereas regarding $A$ we know that for every $B \in \mathcal{A}$ and $a \in A$ the set $a B=L_{a} B$ is in $\mathcal{A}$ but typically will not be equal to $B$ ).

Consider the group

$$
H_{\mathcal{A}}=\left\{h \in G: \text { for every } B \in \mathcal{A}, \quad m_{G}\left(R_{h}(B) \triangle B\right)=0\right\} .
$$

Clearly, $H_{\mathcal{A}}>\Gamma$. We want to reduce to the case that $H_{\mathcal{A}}=\Gamma$.

Lemma 6.1. $H_{\mathcal{A}}$ is a closed subgroup of $G$.

Proof. Indeed, let $m^{\prime}$ be a probability measure in the same measure class as Haar measure $m_{G}$. Then for any $B \in \mathcal{A}$ the characteristic function $1_{B} \in L^{2}\left(m^{\prime}\right)$, and the map $h \mapsto 1_{R_{h}(B)}$ is a continuous map $G \rightarrow L^{2}\left(m^{\prime}\right)$. It follows that $\left\{h: m_{G}\left(R_{h}(B) \triangle B\right)=0\right\}$ is closed and therefore so is $H_{\mathcal{A}}$.

Lemma 6.2. The connected component of the identity $H_{\mathcal{A}}^{0} \leq H_{\mathcal{A}}$ is normal in $G$. 
Proof. From Lemma 4.2 it follows that $G$ is generated by unipotent one parameter subgroups, indeed that there is some element $\alpha^{\mathbf{t}}$ so that $G$ is generated by the groups $U^{\lambda}$ with $\lambda(\mathbf{t}) \neq 0$.

By [22, Theorem II.2.5], for any continuous representation of $G$ any $\Gamma$-invariant subspace is $G$-invariant. Since $\Gamma<H_{\mathcal{A}}$ it follows that the Lie algebra of $H_{\mathcal{A}}^{0}$ is a $\Gamma$-invariant subspace of $\mathfrak{g}$ under the adjoint representation, hence is $G$-invariant. From this we conclude that $H_{\mathcal{A}}^{0}$ is normal in $G$ as claimed.

Now let $N_{\mathcal{A}}$ denote the maximal closed normal subgroup of $G$ contained in $H_{\mathcal{A}}$. By replacing $G$ with $G_{1}=G / N_{\mathcal{A}}$ and $\Gamma$ with the lattice $\Gamma_{1}=H_{\mathcal{A}} / N_{\mathcal{A}}<G_{1}$ we may, and will for the remainder of this section, assume without loss of generality that

$$
H_{\mathcal{A}}=\Gamma \text { and } \Gamma \text { contains no normal subgroup of } G \text {. }
$$

Note that $H_{\mathcal{A}} / N_{\mathcal{A}}$ is discrete since $N_{\mathcal{A}}>H_{\mathcal{A}}^{0}$ by Lemma 6.2.

The following reformulation of (6.1) will be useful: we will say that two points $x, x^{\prime} \in X$ are equivalent modulo $\mathcal{A}\left(x \stackrel{\mathcal{A}}{\sim} x^{\prime}\right)$ if $[x]_{\mathcal{A}}=$ $\left[x^{\prime}\right]_{\mathcal{A}}$. Then since $\mathcal{A}$ is countably generated, (6.1) is easily seen to be equivalent to

$$
H_{\mathcal{A}}=\{h \in G: g h \stackrel{\mathcal{A}}{\sim} g \text { for a.e. } g \in G\} .
$$

Note that the equivalence relation $\stackrel{\mathcal{A}}{\sim}$ induces an equivalence relation on $G$ which we shall also denote by $\stackrel{\mathcal{A}}{\sim}$.

Now consider the group

$$
\begin{aligned}
\Xi & =\left\{\xi \in C_{\mathrm{Aff} G}\left(L_{A}\right): \text { for every } B \in \mathcal{A}, \quad m(\xi(B) \triangle B)=0\right\} \\
& =\left\{\xi \in C_{\mathrm{Aff} G}\left(L_{A}\right): \xi(g) \stackrel{\mathcal{A}}{\sim} g \quad \text { a.e. }\right\},
\end{aligned}
$$

where $C_{\text {Aff } G}\left(L_{A}\right)$ denotes the centralizer in Aff $G$ of the group of left translations $L_{A}$. Notice that by (6.2), the group $\Xi \cap R_{G}=R_{\Gamma}$. The same proof as in Lemma 6.1 gives that $\Xi$ is a closed subgroup of Aff $G$, and hence so is $\Xi_{L}=\Xi \cap L_{G}$. Notice that both $\Xi_{L}$ and $R_{\Gamma}$ are normal subgroups of $\Xi$. We also note the following:

Lemma 6.3. Let $\xi=L_{g_{0}} \xi_{0} \in \Xi$ with $\xi_{0}$ an automorphism of $G$. Then $\xi_{0}(\Gamma)=\Gamma$.

Proof. Suppose for example $\xi_{0}(\Gamma) \not \subset$. Then for almost every $g \in G$ and $\gamma \in \Gamma$ we have

$$
g \xi_{0}(\gamma) \stackrel{\mathcal{A}}{\sim} g_{0}^{-1} \xi_{0}^{-1}(g) \gamma \stackrel{\mathcal{A}}{\sim} g_{0}^{-1} \xi_{0}^{-1}(g) \stackrel{\mathcal{A}}{\sim} g
$$


and $H_{\mathcal{A}} \geq \xi_{0}(\Gamma)$ in contradiction to our assumption. Similarly, one rules out $\xi_{0}(\Gamma) \ngtr \Gamma$ using $\xi_{0}^{-1}$ instead of $\xi_{0}$ in the above argument.

In particular, since $\Gamma$ is a lattice in $G$ we have that

$$
\Xi \leq \operatorname{Aff}_{1} G \text {. }
$$

We recall the definition of the relatively independent joining $m \times{ }_{\mathcal{A}} m$ : it is the measure on $G / \Gamma \times G / \Gamma$ defined by

$$
m \times \times_{\mathcal{A}} m(B \times C)=\int_{X} m_{x}^{\mathcal{A}}(B) \times m_{x}^{\mathcal{A}}(C) d m(x) .
$$

Lemma 6.4. For every $\xi \in \Xi$, the map $\mathrm{Id} \times \xi$ preserves $m \times_{\mathcal{A}} m$.

Proof. Let $\xi \in \Xi$. By definition of $\Xi, \xi$ preserves the atoms of $\mathcal{A}$, so $m$-a.s. one has $m_{\xi(x)}^{\mathcal{A}}=m_{x}^{\mathcal{A}}$. Also, the measure $m$ is preserved by any element of $\operatorname{Aff}_{1} G$, in particular by $\xi$, and so $m$-a.s. $\xi_{*} m_{x}^{\mathcal{A}}=m_{\xi(x)}^{\mathcal{A}}$. Combining this with the previous assertion we get that $\xi_{*} m_{x}^{\mathcal{A}}=m_{x}^{\mathcal{A}}$ a.s. Lemma 6.4 now follows directly from the definition (6.4) of $m \times{ }_{\mathcal{A}} m$.

The results so far are true for any action; now comes the two main steps which comes from careful analysis of the ergodic decomposition of the relatively independent joining $m \times_{\mathcal{A}} m$.

Lemma 6.5. Let $\mathcal{J}_{\mathcal{A}}$ be the set of self joinings $\rho$ for the action corresponding to $\alpha$ on $(X, m)$ (in particular, $\rho$ is a probability measure on $X \times X)$ so that

$$
\rho\left(\left\{\left(x, x^{\prime}\right) \in X \times X: x \stackrel{\mathcal{A}}{\sim} x^{\prime}\right\}\right)=1 .
$$

then for every ergodic $\rho \in \mathcal{J}_{\mathcal{A}}$ there is a $\xi \in \Xi$ (unique up to an element of $\left.R_{\Gamma}\right)$, commuting with $L_{A}$, so that $\rho$ is the unique self joining of $X$ supported on the graph $\{(g, \xi g): g \in G\}$.

Proof. By Theorem 1.4 there is a closed connected subgroup $H \leq G \times$ $G$, projecting onto $G$ in each component, so that $\rho$ is the Haar measure on a closed orbit of $H$. Let $\pi_{1}, \pi_{2}$ denote the projections of $G \times G$ to the first and second coordinate. Suppose this $H$ orbit is $H\left(g_{0}^{\prime}, g_{0}\right)(\Gamma \times \Gamma)=$ $\left(g_{0}^{\prime}, g_{0}\right) \tilde{H}(\Gamma \times \Gamma)$ with $\tilde{H}=\left(g_{0}^{\prime-1}, g_{0}^{-1}\right) H\left(g_{0}^{\prime}, g_{0}\right)$. Since $\pi_{1}(H)=G$, we may as well assume $g_{0}^{\prime}$ is the identity $e \in G$.

Consider now the group $H_{1}=\pi_{2}^{-1}(e) \cap \tilde{H}$. Since $\pi_{1}(H)=G$, and $H$ normalizes $H_{1}$, it follows that $\pi_{1}\left(H_{1}\right) \triangleleft G$. We also claim that $H_{1} \leq H_{\mathcal{A}}$; indeed, for every $h \in \pi_{1}\left(H_{1}\right)$, for almost every $\left(g_{1}, g_{2}\right) \in \tilde{H}$, both $g_{1} \stackrel{\mathcal{A}}{\sim} g_{0} g_{2}$ and $g_{1} h \stackrel{\mathcal{A}}{\sim} g_{0} g_{2}$ hence by transitivity of $\stackrel{\mathcal{A}}{\sim}$ and the fact that $\pi_{1}(H)=G$ it holds that $g_{1} \stackrel{\mathcal{A}}{\sim} g_{1} h$ for almost every $g_{1}$ and so $h \in H_{\mathcal{A}}$. By our assumptions (6.2) on $H_{\mathcal{A}}$ it follows that $\pi_{1}\left(H_{1}\right)$ being 
a normal subgroup of $H_{\mathcal{A}}=R_{\Gamma}$ is the identity, and the same holds for $H_{1}$. Similarly, $H_{2}=\pi_{1}^{-1}(e) \cap \tilde{H}$ is trivial.

It follows that the group $\tilde{H}<G \times G$ is the graph of an automorphism $\xi_{0}$ of $G$ and that for a.e. $g, g \stackrel{\mathcal{A}}{\sim} g_{0} \xi_{0}(g)$, and define $\xi=L_{g_{0}} \xi_{0}$. since $\rho$ is invariant under $\left\{\left(\alpha^{\mathbf{t}}, \alpha^{\mathbf{t}}\right): \mathbf{t} \in \mathbb{R}^{n}\right\}$, this group is contained in $H$, and working through the definitions this implies that $\xi$ commutes with $L_{\alpha^{\mathrm{t}}}$ for every $\mathbf{t}$.

It follows that $\xi \in \Xi$ and that $\rho$ is the unique self joining of $X$ supported on the graph $\{(g \Gamma, \xi g \Gamma): g \in G\}$.

Lemma 6.6. (1) The group $\Phi=\Xi / R_{\Gamma}$ has a $\Phi$-invariant probability measure, hence is compact.

(2) There is a conull set $X_{1} \subset G / \Gamma$ so that for every $x, y \in X_{1}$ in the same atom of $\mathcal{A}$, there is an element $\phi \in \Phi$ taking $x$ to $y$.

Proof. Aff $G$, hence also its closed subgroup $\Xi$, is a locally compact group, $R_{\Gamma}$ a countable discrete subgroup, and so $\Phi=\Xi / R_{\Gamma}$ is a nice locally compact topological group. Note that $\Phi$ acts on $X=G / \Gamma$ by $\left(\xi R_{\Gamma}\right) \cdot(g \Gamma)=\xi(g) \Gamma$ which is well defined by Lemma 6.3 and the definition of $R_{\Gamma}$.

The set $\mathcal{J}_{\mathcal{A}}$ is a Borel subset of the space of probability measures on $X \times X$ equipped with the weak* topology. For any $\xi \in \Xi$, the image $\rho_{\xi}$ of Haar measure on $G / \Gamma$ under $g \Gamma \mapsto g \Gamma, \xi(g) \Gamma$ is an element of $\mathcal{J}_{\mathcal{A}}$, and this map $\xi \mapsto \rho_{\xi}$ is a Borel (indeed, continuous) map $\Xi \rightarrow \mathcal{J}_{\mathcal{A}}$. Since this map is $R_{\Gamma}$-invariant, we actually get a Borel map $\iota: \Phi \rightarrow \mathcal{J}_{\mathcal{A}}$. This map is clearly 1-1, and it follows that the image of $\iota$ is a Borel set and $\iota^{-1}$ is a Borel map [1, Theorem 3.3.2]. ${ }^{4}$

The ergodic decomposition of $m \times{ }_{\mathcal{A}} m$ with respect to the diagonal embedding of the group $A$ gives us a probability measure $\nu$ on the space of joinings of $X$ with itself. Since by definition

$$
m \times{ }_{\mathcal{A}} m\left(\left\{\left(x, x^{\prime}\right) \in X \times X: x \stackrel{\mathcal{A}}{\sim} x^{\prime}\right\}\right)=1
$$

the same holds for almost every ergodic component of $m \times_{\mathcal{A}} m$, hence $\nu$ is supported on the ergodic joinings in $\mathcal{J}_{\mathcal{A}}$, which by Lemma 6.5 are in $\iota(\Phi)$. Using $\iota^{-1}$, we get a Borel probability measure $\nu^{\prime}=\iota_{*}^{-1} \nu$ on $\Phi$.

By Lemma 6.4, for every $\xi \in \Xi$, we have that $(\mathrm{Id}, \xi)$ preserves $m \times_{\mathcal{A}}$ $m$, and since $\xi$ commutes with $L_{A}$ it follows that $(\mathrm{Id}, \xi)_{*}$ preserves $\nu$.

\footnotetext{
${ }^{4}$ This whole discussion (which is somewhat less generally applicable than the rest of this proof) can be circumvented by defining an associative composition operation on $\mathcal{J}_{\mathcal{A}}$ which can be shown to give a group structure when restricted to the ergodic joinings, or, what amounts to the same, by using the topology of convergence in measure on $\operatorname{Aff}(G / \Gamma)$. See [35] for further discussion of this issue.
} 
But for every $\rho_{\xi^{\prime}} \in \mathcal{J}_{\mathcal{A}}$,

$$
(\mathrm{Id}, \xi)_{*}\left(\rho_{\xi^{\prime}}\right)=\rho_{\xi \xi^{\prime}}
$$

and so $\nu^{\prime}$ is right invariant under the action of $\Xi$ on $\Phi$. It follows that $\nu^{\prime}$ is a finite Haar measure on the locally compact group $\Phi$, hence $\Phi$ is a compact group, proving Lemma 6.6.(1).

To see (2), we first present $m \times_{\mathcal{A}} m$ in two ways: for any bounded measurable $f$,

$$
\begin{aligned}
\int_{X \times X} f(x, y) d m \times_{\mathcal{A}} m(x, y) & =\iint_{X} f(x, y) d m_{x}^{\mathcal{A}}(y) d m(x) \\
& =\int_{X \times X} \int_{\mathcal{J}_{\mathcal{A}}} f(x, y) d \rho(x, y) d \nu(\rho) \\
& =\int_{X} \int_{\Phi} f(x, \phi(x)) d x d \nu^{\prime}(\phi) .
\end{aligned}
$$

It follows that $m$-a.s. $m_{x}^{\mathcal{A}}=\left(\tilde{R}_{x}\right)_{*}\left(\nu^{\prime}\right)$, with $\tilde{R}_{x}: \Phi \rightarrow X$ the map $\phi \mapsto \phi(x)$.

Let $X_{1}$ denote the set

$$
X_{1}=\left\{x \in X: m_{x}^{\mathcal{A}}=\left(\tilde{R}_{x}\right)_{*}\left(\nu^{\prime}\right)\right\} .
$$

Suppose $x, x^{\prime} \in X_{1}$ with $x \stackrel{\mathcal{A}}{\sim} x^{\prime}$, i.e. $[x]_{\mathcal{A}}=\left[x^{\prime}\right]_{\mathcal{A}}$ and hence $m_{x}^{\mathcal{A}}=m_{x^{\prime}}^{\mathcal{A}}$. Then $m_{x}^{\mathcal{A}}$-almost every $y \in[x]_{\mathcal{A}}$ can be represented as $\eta(x)$ as well as $\phi\left(x^{\prime}\right)$ for $\eta, \phi \in \Xi / R_{\Gamma}$. Find one $y \in[x]_{\mathcal{A}}$ that can be represented in both of these ways. Then $x=\eta^{-1} \phi\left(x^{\prime}\right)$ with $\eta^{-1} \phi \in \Phi$ as claimed.

Corollary 6.7. The sigma algebra $\mathcal{B}_{\Phi}$ of $\Phi$-invariant Borel subsets of $X$ is countably generated and is equivalent $\bmod m$ to $\mathcal{A}$.

Proof. That $\mathcal{B}_{\Phi}$ is countably generated is a general property of compact group actions on standard Borel spaces, which can be easily verified as follows: take $B_{i}$ to be a generating sequence of Borel sets in $X$. Consider the functions $f_{B_{i}}=\int_{\Phi} 1_{B_{i}}(\phi x) d \phi$. The level sets $\left\{a<f_{B_{i}}(x)<b\right\}$ for $a, b \in \mathbb{Q}$ form a countable generating set for $\mathcal{B}_{\Phi}$.

We now show that $\mathcal{B}_{\Phi}$ is equivalent $\bmod m$ to $\mathcal{A}$. By the definition (6.3) of $\Xi$, every set $B \in \mathcal{A}$ is almost invariant under $\Xi$ and therefore also under $\Phi$, i.e. $m(\phi B \triangle B)=0$ for every $\phi \in \Phi$. As is well-known (see e.g. [36, Lemma 2.2.15]) this implies that there is a $\Phi$-invariant Borel set $B^{\prime}$ with $m\left(B^{\prime} \triangle B\right)=0$ and by definition $B^{\prime} \in \mathcal{B}_{\Phi}$.

Conversely, let $B \in \mathcal{B}_{\Phi}$. Then the function

$$
\mathbf{E}\left(1_{B} \mid \mathcal{A}\right)(x)=\int_{X} 1_{B}\left(x^{\prime}\right) d m_{x}^{\mathcal{A}}\left(x^{\prime}\right)
$$


is $\mathcal{A}$-measurable. Let $X_{1}$ be a conull set as in Lemma 6.6.(2). For every $x \in B \cap X_{1}$ we have that $B \supset \Phi x \supset[x]_{\mathcal{A}} \cap X_{1}$, hence

$$
m_{x}^{\mathcal{A}}(B) \geq m_{x}^{\mathcal{A}}\left([x]_{\mathcal{A}} \cap X_{1}\right)=1 \quad \text { a.s }
$$

and hence $\mathbf{E}\left(1_{B} \mid \mathcal{A}\right) \geq 1_{B}$ a.e.. Applying the same to $B^{\complement}$ we get the reverse inequality, hence $\mathbf{E}\left(1_{B} \mid \mathcal{A}\right)=1_{B}$ a.e. and so there is a $B^{\prime} \in \mathcal{A}$ with $m\left(B \triangle B^{\prime}\right)=0$.

This completes the proof of Theorem 1.5. We now specialize to the case where $G$ is semisimple with finite center, all factors have $\mathbb{R}$-rank $\geq 2$, and $\Gamma<G$ is an irreducible lattice (i.e. a lattice which is not commensurable to a product of lattices in factors of $G$ ) and deduce Corollary 1.6 from the more general Theorem 1.5. As in that corollary, we let $A=\alpha\left(\mathbb{R}^{n}\right)$ to be the connected component of the identity in a maximal $\mathbb{R}$-split torus. Then its centralizer $C_{G}(A)=M A$ where $M=C_{K}(A)$ by $[17$, Lemma 7.22] and the fact that $A$ is maximal $\mathbb{R}$ split. Here $M$ is the unique maximal compact subgroup of $C_{G}(A)$, it is compact since $K$ is compact by the assumption that $G$ has finite center, see [17, Theorem 6.31]. Note that in this case, since $\Gamma$ is irreducible, there can be no nontrivial connected $N \triangleleft G$ so that $N \Gamma$ is closed, hence $\Gamma^{\prime}:=H_{\mathcal{A}} \geq \Gamma$ is discrete.

Lemma 6.8. Let $G, \Gamma, \alpha$ and $\mathcal{A}$ be as in Corollary 1.6 (with $\mathcal{A}$ identified with a $\sigma$-algebra of left $M$-invariant subsets of $G / \Gamma$ in the obvious way $\left.{ }^{5}\right)$. Then

(1) there is an open neighborhood $\mathcal{U}$ of the identity in $\operatorname{Aff}_{1}(G)$ so that $\mathcal{U} \cap \Xi \subset L_{G}$,

(2) $\Xi \cap L_{G}=L_{M}$

Proof. Claim (1) follows from the fact that $\Gamma^{\prime}$ is finitely generated and Lemma 6.3.

Since $\mathcal{A}$ consists of $L_{M}$-invariant sets by definition of $\Xi, L_{M} \leq$ $L_{G} \cap \Xi$. By definition of $\Xi$, every $\xi \in \Xi$ commutes with left translation by elements of $A$, so $\Xi \cap L_{G} \leq L_{C_{G}(A)}$. Recall that $C_{G}(A)=M A$. If $\Xi \cap L_{G}>L_{M}$ holds strictly, then $\Xi \cap L_{G}$ contains a nontrivial element of $L_{A}$. However, this is impossible since $\Phi=\Xi / R_{\Gamma^{\prime}} \leq \operatorname{Aff}_{1}(G / \Gamma)$ is compact and since the powers of a nontrivial element of $L_{A}$ are not contained in a compact subset of $\operatorname{Aff}_{1}(G / \Gamma)$.

Proof of Corollary 1.6. By Lemma $6.8 L_{M} \triangleleft \Xi$ and $\Xi / L_{M}$ is discrete, so $\Xi / L_{M} R_{\Gamma^{\prime}}$ is both discrete and compact, hence finite, and consequently

\footnotetext{
${ }^{5}$ If we view $\mathcal{A}$ in this way then the assertion of Corollary 1.6 that $[y]_{\mathcal{A}}$ is finite for a.e. $y \in M \backslash G / \Gamma$ translates to $[x]_{\mathcal{A}}$ is a union of finitely many orbits of $M$ on $G / \Gamma$.
} 
so is $\Xi / L_{M} R_{\Gamma}$. By Lemma 6.6.(2) this shows that for a.e. $x \in G / \Gamma$ the atom $[x]_{\mathcal{A}}$ consists of finitely many orbits of $M$. This concludes the proof of the first claim in the corollary.

If $\Gamma$ is maximal, $\Gamma^{\prime}=\Gamma$ and furthermore the normalizer in $G$ of $\Gamma$ is equal to $\Gamma$. Any $\xi \in \Xi$ can be written as $\xi=L_{h} \xi_{0}=\xi_{1} R_{h^{\prime}}$ with $\xi_{0}, \xi_{1} \in \operatorname{Aut} G$; by definition of $\Xi, \xi_{1}$ fixes $A_{0}$ pointwise, and by Lemma $6.3 \xi_{0}$ sends $\Gamma$ to itself. By the assumptions on $G$ and $A$ in the second part of the corollary, $\xi_{1}$ and hence also $\xi_{0}$ is necessarily an inner automorphism, and by the maximality of $\Gamma$ we have that $\xi_{0}(g)=\gamma^{-1} g \gamma$ for some $\gamma \in \Gamma$. It follows that $L_{h \gamma^{-1}} \in \Xi$ and so by Lemma 6.8.(2) $h \gamma^{-1} \in M$, and $\xi \in L_{M} R_{\Gamma}$. It follows that $[x]_{\mathcal{A}}$ is almost surely a single orbit of $M$, or translating back to $y \in M \backslash G / \Gamma$ that $[y]_{\mathcal{A}}$ is a single point a.s.: which implies that when viewing $\mathcal{A}$ as a $\sigma$-algebra of Borel subsets of $M \backslash G / \Gamma$ that it is equal mod $m$ to the full Borel sigma algebra.

\section{EQUIDISTRIBUTION}

As pointed out in Section 1 Theorem 1.1 follows from Theorem 1.4. We now give the proof of the corollary regarding equidistributed orbits.

Proof of Corollary 1.2. In the following write $\alpha_{i}$ for the action on $X_{i}=$ $G / \Gamma_{i}$ for $i=1,2$ and $\alpha$ for the action on $X_{1} \times X_{2}$. Let $x=\left(x_{1}, x_{2}\right)$ be as in the corollary. For $\ell>0$ define the probability measure

$$
\mu_{\ell}(B)=\frac{1}{2^{n} \ell^{n}} \int_{[-\ell, \ell]^{n}} 1_{B}\left(\alpha^{\mathbf{t}} x\right) \mathrm{d} \mathbf{t}
$$

for any measurable $B \subset X$. Then a weak* limit $\mu$ of any subsequence of $\mu_{\ell}$ for $\ell \rightarrow \infty$ is $\alpha$-invariant. Moreover, we claim $\mu$ is a probability measure and a joining between $\alpha_{1}$ and $\alpha_{2}$. However, then the assumptions to the corollary and Theorem 1.1 show that $\mu=m_{1} \times m_{2}$ is the only such measure. Since $\mu$ was any weak* limit, the corollary follows.

To see the claim, let $f \in C\left(X_{1}\right)$ be non-negative with compact support. Let $\epsilon>0$ be arbitrary, take a $g \in C\left(X_{2}\right)$ with $0 \leq g \leq 1$, compact support, and $\int g \mathrm{~d} m_{1}>1-\epsilon$. Then for large enough $\ell$ we have

$$
\int_{[-\ell, \ell]^{n}} g\left(\alpha_{2}^{\mathbf{t}}\left(x_{2}\right)\right) \mathrm{d} \mathbf{t}>(1-\epsilon) 2^{n} \ell^{n}
$$


by the assumption that $x_{2}$ has $m_{2}$-equidistributed $\alpha_{2}$-orbit. Therefore,

$$
\begin{gathered}
\frac{1}{2^{n} \ell^{n}} \int_{[-\ell, \ell]^{n}} f\left(\alpha_{1}^{\mathbf{t}} x_{1}\right) \mathrm{d} \mathbf{t} \leq \frac{1}{2^{n} \ell^{n}} \int_{[-\ell, \ell]^{n}} f \otimes g\left(\alpha^{\mathbf{t}}\left(x_{1}, x_{2}\right)\right) \mathrm{d} \mathbf{t}+\epsilon\|f\|_{\infty} \leq \\
\frac{1}{2^{n} \ell^{n}} \int_{[-\ell, \ell]^{n}} f\left(\alpha_{1}^{\mathbf{t}} x_{1}\right) \mathrm{d} \mathbf{t}+\epsilon\|f\|_{\infty}
\end{gathered}
$$

which shows that

$$
\left|\int f \otimes g \mathrm{~d} \mu-\int f \mathrm{~d} m_{1}\right| \leq \epsilon\|f\|_{\infty} .
$$

Since this holds for any $g$ with compact support as above and any $\epsilon$, we conclude that

$$
\int f \mathrm{~d}\left(\pi_{1}\right)_{*} \mu=\int f \mathrm{~d} m_{1} .
$$

Varying $f$ the claim and the corollary follow.

\section{ACKNOWLEDGEMENTS}

We thank Michael Larsen, Dave Witte Morris, and Akshay Venkatesh for helpful discussions.

\section{REFERENCES}

[1] William Arveson. An invitation to $C^{*}$-algebras. Springer-Verlag, New York, 1976. Graduate Texts in Mathematics, No. 39.

[2] S. G. Dani. Bernoullian translations and minimal horospheres on homogeneous spaces. J. Indian Math. Soc. (N.S.), 40(1-4):245-284 (1977), 1976.

[3] A. del Junco and D. Rudolph. On ergodic actions whose self-joinings are graphs. Ergodic Theory Dynam. Systems, 7(4):531-557, 1987.

[4] M. Einsiedler and A. Katok. Rigidity of measures - the high entropy case, and non-commuting foliations. to appear in Israel J. Math.

[5] M. Einsiedler and A. Katok. Invariant measures on $G / \Gamma$ for split simple Liegroups G. Comm. Pure Appl. Math., 56(8):1184-1221, 2003.

[6] M. Einsiedler, A. Katok, and E. Lindenstrauss. Invariant measures and the set of exceptions to Littlewood's conjecture. to appear in Ann. of Math.

[7] M. Einsiedler and E. Lindenstrauss. Rigidity properties of $\mathbb{Z}^{d}$-actions on tori and solenoids. Electron. Res. Announc. Amer. Math. Soc., 9:99-110, 2003.

[8] M. Einsiedler and T. Ward. Entropy geometry and disjointness for zerodimensional algebraic actions. to appear in Crelles Journal, 21 pages.

[9] Harry Furstenberg. Disjointness in ergodic theory, minimal sets, and a problem in Diophantine approximation. Math. Systems Theory, 1:1-49, 1967.

[10] E. Glasner. Ergodic theory via joinings, volume 101 of Mathematical Surveys and Monographs. American Mathematical Society, Providence, RI, 2003.

[11] B. Hasselblatt and A. Katok. Principal structures. In Handbook of dynamical systems, Vol. 1A, pages 1-203. North-Holland, Amsterdam, 2002.

[12] B. Kalinin and R. Spatzier. Measurable rigidity for higher rank abelian actions. to appear in Ergod. Th. Dynam. Sys. 
[13] Boris Kalinin and Anatole Katok. Measurable rigidity and disjointness for $\mathbb{Z}^{k}$ actions by toral automorphisms. Ergodic Theory Dynam. Systems, 22(2):507$523,2002$.

[14] A. Katok and R. J. Spatzier. Invariant measures for higher-rank hyperbolic abelian actions. Ergodic Theory Dynam. Systems, 16(4):751-778, 1996.

[15] A. Katok and R. J. Spatzier. Corrections to: "Invariant measures for higherrank hyperbolic abelian actions" [Ergodic Theory Dynam. Systems 16 (1996), no. 4, 751-778; MR 97d:58116]. Ergodic Theory Dynam. Systems, 18(2):503$507,1998$.

[16] D. Kleinbock, N. Shah, and A. Starkov. Dynamics of subgroup actions on homogeneous spaces of Lie groups and applications to number theory. In Handbook of dynamical systems, Vol. 1A, pages 813-930. North-Holland, Amsterdam, 2002.

[17] Anthony W. Knapp. Lie groups beyond an introduction, volume 140 of Progress in Mathematics. Birkhäuser Boston Inc., Boston, MA, second edition, 2002.

[18] F. Ledrappier and L.-S. Young. The metric entropy of diffeomorphisms. I. Characterization of measures satisfying Pesin's entropy formula. Ann. of Math. (2), 122(3):509-539, 1985.

[19] F. Ledrappier and L.-S. Young. The metric entropy of diffeomorphisms. II. Relations between entropy, exponents and dimension. Ann. of Math. (2), 122(3):540-574, 1985.

[20] Elon Lindenstrauss. Invariant measures and arithmetic quantum unique ergodicity. to appear in Ann. of Math.

[21] Elon Lindenstrauss. Rigidity of multiparameter actions. preprint (18 pages), 2003.

[22] G. A. Margulis. Discrete subgroups of semisimple Lie groups, volume 17 of Ergebnisse der Mathematik und ihrer Grenzgebiete (3) [Results in Mathematics and Related Areas (3)]. Springer-Verlag, Berlin, 1991.

[23] G. A. Margulis and G. M. Tomanov. Invariant measures for actions of unipotent groups over local fields on homogeneous spaces. Invent. Math., 116(1-3):347$392,1994$.

[24] Gregory Margulis. Problems and conjectures in rigidity theory. In Mathematics: frontiers and perspectives, pages 161-174. Amer. Math. Soc., Providence, RI, 2000.

[25] Calvin C. Moore. Ergodicitiy of flows on homogeneous spaces. Amer. J. Math., 88:154-178, 1966.

[26] William Parry. Topics in ergodic theory, volume 75 of Cambridge Tracts in Mathematics. Cambridge University Press, Cambridge, 1981.

[27] Marina Ratner. Horocycle flows, joinings and rigidity of products. Ann. of Math. (2), 118(2):277-313, 1983.

[28] Marina Ratner. On Raghunathan's measure conjecture. Ann. of Math. (2), 134(3):545-607, 1991.

[29] Daniel J. Rudolph. $\times 2$ and $\times 3$ invariant measures and entropy. Ergodic Theory Dynam. Systems, 10(2):395-406, 1990.

[30] Ja. G. Sină. A weak isomorphism of transformations with invariant measure. Dokl. Akad. Nauk SSSR, 147:797-800, 1962. 
[31] Alexander N. Starkov. Dynamical systems on homogeneous spaces, volume 190 of Translations of Mathematical Monographs. American Mathematical Society, Providence, RI, 2000. Translated from the 1999 Russian original by the author.

[32] V. S. Varadarajan. Groups of automorphisms of Borel spaces. Trans. Amer. Math. Soc., 109:191-220, 1963.

[33] V. S. Varadarajan. Lie groups, Lie algebras, and their representations. Prentice-Hall Inc., Englewood Cliffs, N.J., 1974. Prentice-Hall Series in Modern Analysis.

[34] Dave Witte. Measurable quotients of unipotent translations on homogeneous spaces. Trans. Amer. Math. Soc., 345(2):577-594, 1994.

[35] Dave Witte. Correction and extension of: "Measurable quotients of unipotent translations on homogeneous spaces" [Trans. Amer. Math. Soc. 345 (1994), no. 2, 577-594]. Trans. Amer. Math. Soc., 349(11):4685-4688, 1997.

[36] Robert J. Zimmer. Ergodic theory and semisimple groups, volume 81 of Monographs in Mathematics. Birkhäuser Verlag, Basel, 1984. 